\title{
The RUNX1/IL-34/CSF-1R axis is an autocrinally regulated modulator of resistance to BRAF-V600E inhibition in melanoma
}

Orsi Giricz, ${ }^{1}$ Yongkai Mo, ${ }^{1}$ Kimberly B. DahIman, ${ }^{2}$ Xiomaris M. Cotto-Rios, ${ }^{3}$ Chiara Vardabasso, ${ }^{4}$ Hoa Nguyen, ${ }^{5}$ Bernice Matusow, ${ }^{5}$ Matthias Bartenstein, ${ }^{1}$ Veronika Polishchuk, ${ }^{1}$ Douglas B. Johnson, ${ }^{2}$ Tushar D. Bhagat, ${ }^{1}$ Rafe Shellooe, ${ }^{5}$ Elizabeth Burton, ${ }^{5}$ James Tsai, ${ }^{5}$ Chao Zhang, ${ }^{5}$ Gaston Habets, ${ }^{5}$ John M. Greally, ${ }^{6}$ Yiting Yu, ${ }^{1}$ Paraic A. Kenny, ${ }^{7}$ Gregg B. Fields, ${ }^{8}$ Kith Pradhan, ${ }^{1}$ E. Richard Stanley, ${ }^{7}$ Emily Bernstein, ${ }^{4}$ Gideon Bollag, ${ }^{5}$ Evripidis Gavathiotis, ${ }^{3}$ Brian L. West, ${ }^{5}$ Jeffrey A. Sosman, ${ }^{2}$ and Amit K. Verma ${ }^{1}$

'Department of Medicine, Albert Einstein College of Medicine, Montefiore Medical Center, Bronx, New York, USA. ${ }^{2}$ Vanderbilt University, Nashville, Tennessee, USA. ${ }^{3}$ Department of Biochemistry, Albert Einstein College of Medicine, Bronx, New York, USA. ${ }^{4}$ Departments of Oncological Sciences \& Dermatology, Icahn School of Medicine at Mount Sinai, New York, New York, USA. ${ }^{5}$ Plexxikon, Berkeley, California, USA. ${ }^{6}$ Department of Genetics and ${ }^{7}$ Department of Developmental and Molecular Biology, Albert Einstein College of Medicine, Bronx, New York, USA. ${ }^{8}$ Department of Chemistry and Biochemistry, Florida Atlantic University, Florida, USA.

Resistance to current therapies still impacts a significant number of melanoma patients and can be regulated by epigenetic alterations. Analysis of global cytosine methylation in a cohort of primary melanomas revealed a pattern of early demethylation associated with overexpression of oncogenic transcripts. Loss of methylation and associated overexpression of the CSF 1 receptor (CSF1R) was seen in a majority of tumors and was driven by an alternative, endogenous viral promoter in a subset of samples. CSF1R was particularly elevated in melanomas with BRAF and other MAPK activating mutations. Furthermore, rebound ERK activation after BRAF inhibition was associated with RUNX1-mediated further upregulation of CSF-1R and its ligand IL-34. Importantly, increased CSF-1R and IL-34 overexpression were detected in an independent cohort of resistant melanomas. Inhibition of CSF-1R kinase or decreased CSF-1R expression by RNAi reduced 3-D growth and invasiveness of melanoma cells. Coinhibition of CSF-1R and BRAF resulted in synergistic efficacy in vivo. To our knowledge, our data unveil a previously unknown role for the autocrine-regulated CSF-1R in BRAF V600E resistance and provide a preclinical rationale for targeting this pathway in melanoma.

Conflict of interest: THN, BM, RS, EB, JT, CZ, CH, CBF, CB, and BLW are employees of Plexxikon.

Submitted: February 8, 2018

Accepted: June 12, 2018

Published: July 25, 2018

\section{Reference information:} JCI Insight. 2018;3(14):e120422. https://doi.org/10.1172/jci. insight.120422.

\section{Introduction}

The incidence of melanoma is on the rise in the US, and when the disease is diagnosed at an advanced stage, it presents a serious challenge in terms of treatment. While the 5-year survival of the primary disease is over $95 \%$, once it disseminates to distant sites, this number drops to less than $15 \%$ (1). Molecular targeted therapy is an important therapeutic option, especially when targeting the MAPK pathway, as it is deregulated in over $90 \%$ of patients (2). The discovery of the constitutively activating BRAF V600E mutation resulted in the clinical development of BRAF inhibitors, showing high rates of rapid response as monotherapy but with a limited duration of response as the majority of patients develop resistance (3). Combination therapy of BRAF inhibitors with MEK inhibitors increases progression-free and overall survival, even delaying the development of resistance to 9.4 months, though most cases eventually relapse (4). Immune checkpoint inhibition is a major advance in melanoma therapeutics, but it is also associated with relapsed or refractory disease in more than $50 \%$ of patients $(5,6)$. Therefore, there is still a critical need to understand mechanisms of resistance to these agents and to find novel therapeutics to further extend survival of these patients, potentially in combination with the currently accepted clinical modalities. 
In an attempt to comprehensively define the molecular alterations in this disease, we performed an integrative global analysis to examine cytosine methylation, gene copy number alterations, and gene expression profiling, as well as selected genetic mutations in untreated melanoma patient samples. We demonstrate that the treatment-naive melanoma genome is characterized by widespread demethylation as the predominant epigenetic change and that this is associated with upregulation of various immune- and cancer-related pathways. The CSF-1 receptor (CSF-1R) was significantly overexpressed and associated with promoter demethylation in melanoma samples. We also observed that the CSF1R is additionally expressed via an alternative viral promoter in this disease. Utilizing RNA interference and a clinically relevant inhibitor, we have found that the CSF-1R contributes to growth and invasion in melanoma. Most importantly, CSF-1R overexpression on malignant melanocytes plays an important role in the ERK activation rebound seen after BRAF inhibitor treatment, and coinhibition of the CSF-1R kinase is efficacious in BRAF V600E melanoma both in vivo and in vitro. Thus, in addition to known immuno-oncogenic effects of CSF-1R in macrophages in the tumor microenvironment, our data illustrate a potentially novel critical oncogenic role of autocrine CSF-1R and IL-34 pathways in melanoma.

\section{Results}

Melanoma is characterized by widespread changes in DNA methylation. Even though mutations in epigenetic regulators and oncogenic pathways are seen in melanoma, their effects on the epigenome are only recently beginning to come under scrutiny, with numerous studies mainly focusing on aberrant methylation of promoter $\mathrm{CpG}$ islands (7-9). We analyzed the methylome of 29 cases of untreated primary and metastatic malignant melanoma (Supplemental Table 1; supplemental material available online with this article; https://doi.org/10.1172/jci. insight.120422DS1) via the HpaII tiny fragment enriched by ligation-mediated-PCR (HELP) assay $(10,11)$ and compared it with primary normal human epidermal melanocytes (NHEM) as controls. Unsupervised clustering revealed that the melanoma samples were epigenetically distinct from controls, distributing them into 3 main clusters: M1, M2, and M3 (Figure 1A). Analysis of cytosine methylation differences indicated that melanoma, as a whole, was characterized by widespread demethylation (Figure 1B), with groups M1, M2, and M3 being characterized by increasing demethylation correlating with the magnitude of their dissimilarity from control melanocytes (Supplemental Figure 1A). The demethylation was validated on a genome-wide level by the luminometric methylation assay (LUMA) and demonstrated significantly less cytosine methylation in the melanoma samples (Figure 1C). Genome-wide loss of methylation was seen in early primary lesions and persisted in nodal and soft-tissue metastasis (Figure 1, D and E). Analyzing 3 cases where we had matched samples from both the primary and metastatic lesions, we observed more hypomethylated loci in metastatic samples when compared with the primary tumor from the same patient (Supplemental Figure 1B). Analysis of regional distribution of the differentially methylated regions demonstrated that even though these changes were present genome wide (Supplemental Figure 3A), there was a regional bias for chromosomes 9,20 and $21(P=0.047,0.001$, and $<0.0001$ respectively) as compared to the genomic distribution of all HpaII fragments from the HELP array (Supplemental Figure 1, C-E). Epigenomic clustering of melanoma was found to be independent of demographics and clinical characteristics such as staging or depth (Supplemental Figure 2A). Parallel gene expression profiling of the same samples also revealed transcriptomic differences between melanomas and controls, which was again independent of demographics and tumor grade and stage (Supplemental Figure 2B).

Next, we evaluated the genomic distribution of aberrantly methylated regions in all melanoma samples and observed that hypomethylated regions were preferentially observed outside of $\mathrm{CpG}$ islands (Figure $1 F)$, consistent with other reports showing non-CpG island changes in cancer (12). Since demethylation has been associated with genomic instability (13), we analyzed copy number alterations in the same samples by array CGH. We observed epigenetic clusters M2 and M3 (Figure 1A) that were more epigenetically dissimilar and demethylated when compared with controls and were characterized by larger numbers of genomic gains and losses, suggesting higher genomic instability (Figure 1G). Array CGH also revealed a set of known and potentially novel deletions (Supplemental Table 2) and amplifications in melanoma (Supplemental Table 3). Correlation between aberrant methylation and gene expression suggested that many important genes were affected by coordinated changes in DNA methylation and expression in melanoma. Ingenuity Pathway Analysis (IPA, QIAGEN Inc., https://www.qiagenbioinformatics.com/products/ingenuitypathway-analysis) revealed immune response, tumorigenesis, cancer, and dermatological disorders to be the top 4 genetic pathways altered in all melanoma samples and demonstrated that aberrant methylation is associated with upregulation of various oncogenic transcripts (Supplemental Table 4). 
A

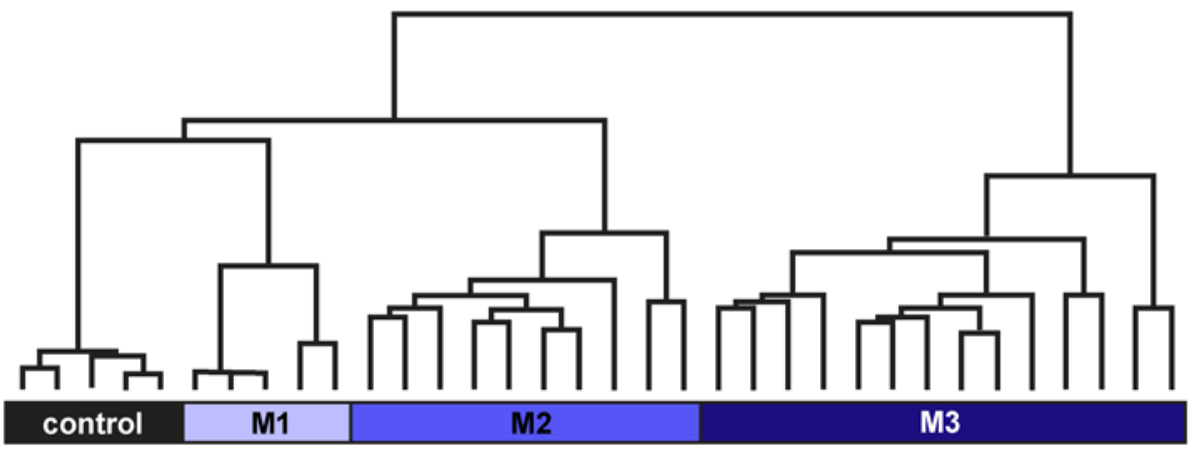

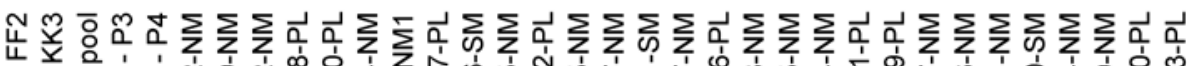

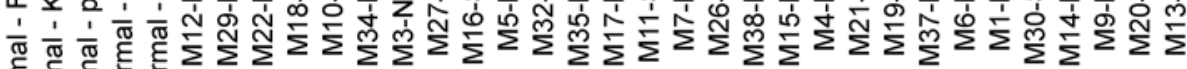

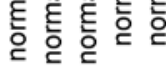

$\mathbf{F}$

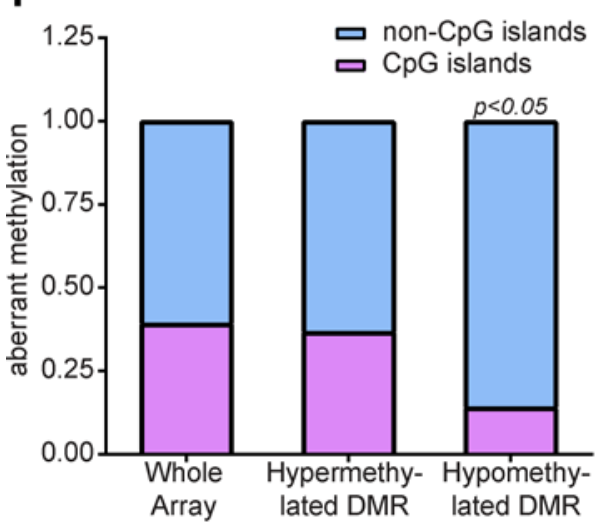

G

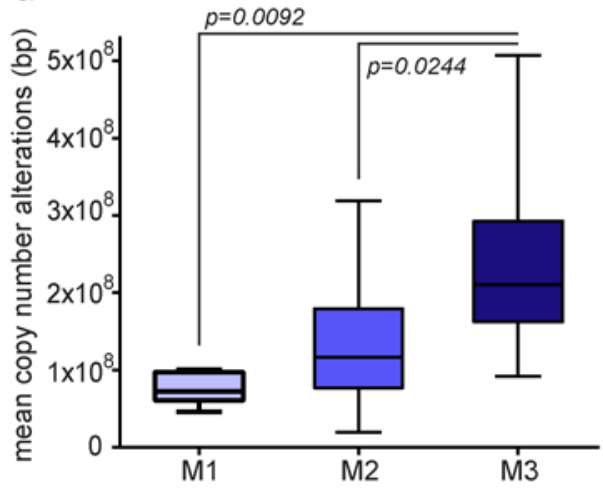

H

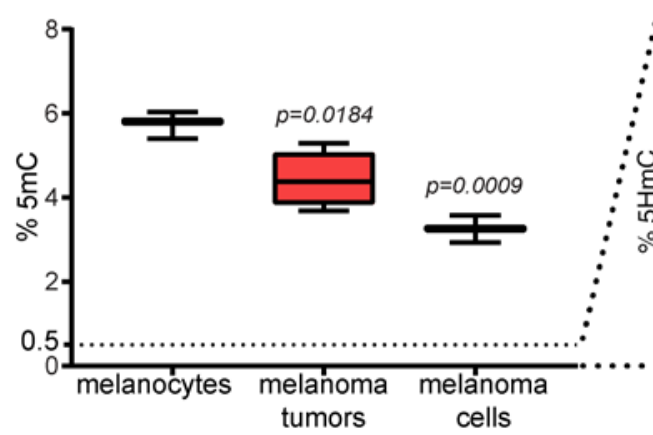

B

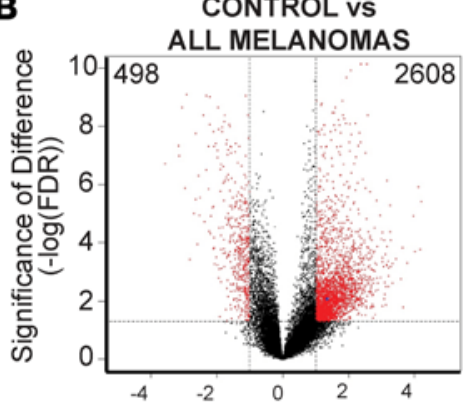

C

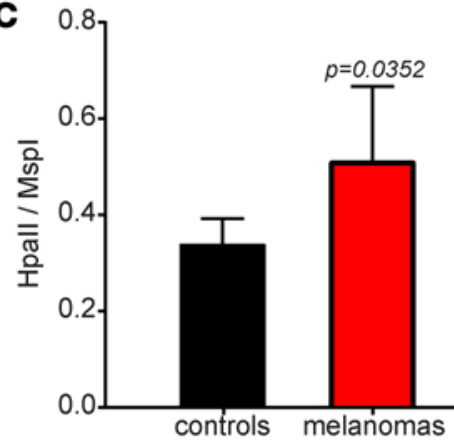

D

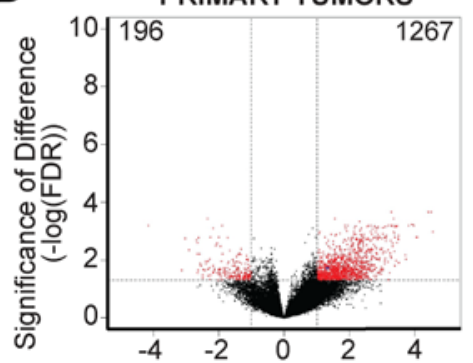

E

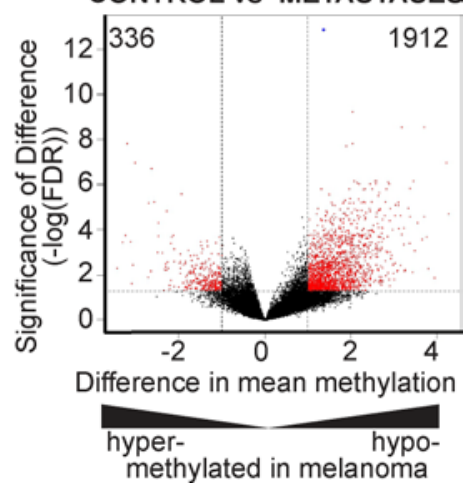

Figure 1. Melanoma is characterized by widespread changes in DNA methylation. (A) Unsupervised hierarchical clustering of DNA methylation profiles generated by the HELP assay. The melanoma samples are separated into 3 major clusters (M1, M2, and M3). PL, primary lesion; NM, nodal metastasis; SM, soft-tissue metastasis. Clinical data are provided in Supplemental Table 1. (B) Volcano plot showing difference of mean methylation and statistical significance of the difference, with the number of differentially methylated loci indicated. Numbers to the left indicate hypermethylated loci, and numbers to the right indicate Differentially methylated regions (DMRs) in melanoma. An FDR of less than $5 \%$ is used as a marker of significant differences. (C) Global methylation levels tested by the LUMA assay indicate loss of methylation in malignant melanoma samples $(n=36)$ (unpaired $t$ test, 2-tailed). ( $\mathbf{D}$ and $\mathbf{E}$ ) Predominantly hypomethylated loci are seen in primary tumors and nodal and distant metastasis. The single blue dot (located in the right upper quadrant) in the volcano plots indicate the CSF1R locus. (F) The genomic position of every Hpall-amplifiable fragment on the HELP array was compared with the location of known CpG islands, demonstrating demethylated DMRs enriched outside of CpG-islands (proportions test). (C) Box plots of mean DNA copy number alterations (gains + losses) for melanoma sample clusters based on methylation (M1, M2, and M3) (1-way ANOVA). (H) Global methylation (\%5mC) and hydroxymethylation $(\% 5 \mathrm{HmC})$ in melanocytes $(n=3)$, melanoma tumors $(n=4)$, and cells $(n=3)(1$-way ANOVA). 


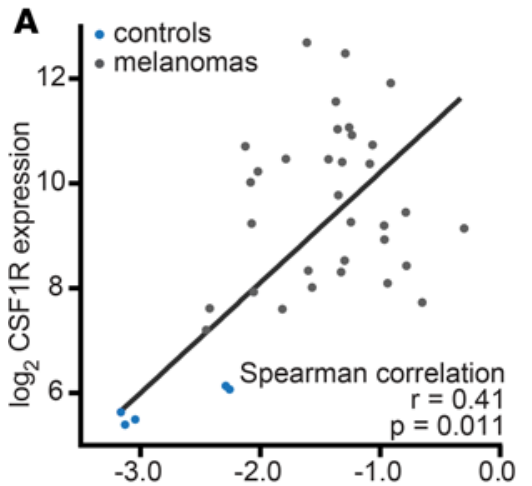

promoter methylation ( $\log _{2}$ [Hpall/Mspl])

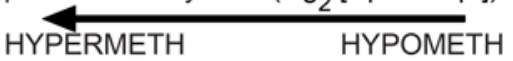

B

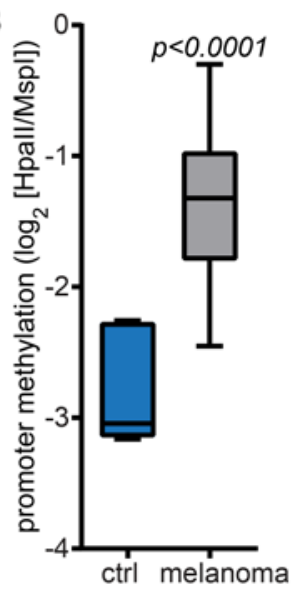

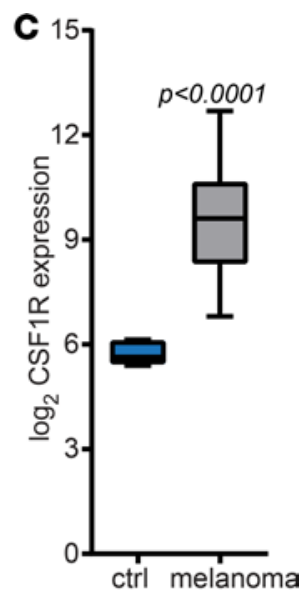

0.0379

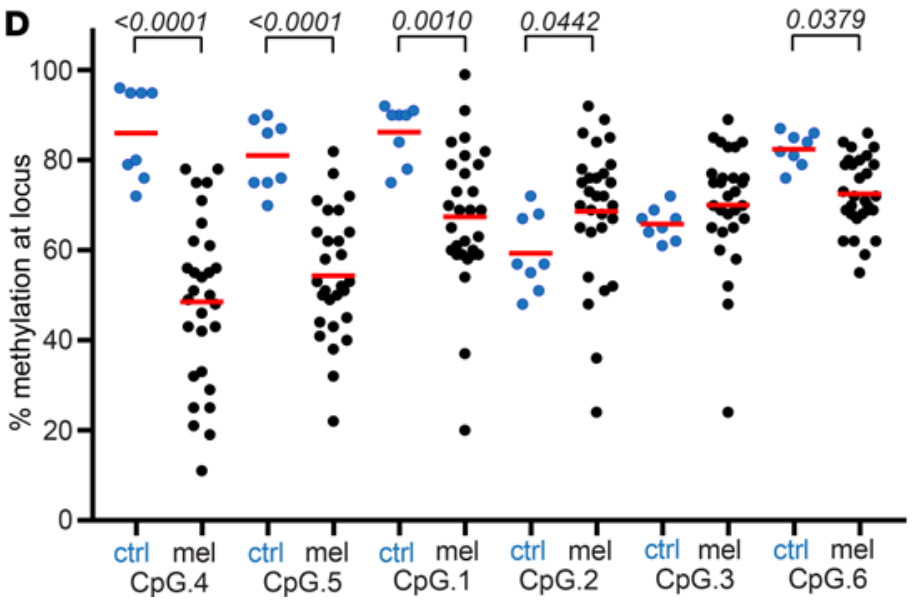

Figure 2. CSF1R is hypomethylated and overexpressed in melanoma in a large subset of samples. (A) Mean hypomethylation of the CSF1R promoter shows positive correlation with gene expression across all samples. The greater value of the $\log _{2}$ (Hpall/ Mspl) demonstrates less methylation (Spearman's correlation, $r=0.41, P=$ 0.011). (B) Mean methylation of the CSF1R promoter in melanoma samples compared with melanocyte controls shows less methylation in melanoma (unpaired $t$ test, 2 -tailed; $P<0.0001$ ). (C) Mean array-based expression of CSF1R in melanoma samples compared with melanocyte controls $(n=5)$ shows higher expression in melanoma $(n=36)$ (unpaired $t$ test, 2-tailed; $P<0.0001$ ). (D) MassARRAY-based single bp validation of $\mathrm{CpG}$ methylation (CpG.1 through $C p G .6)$ within the CSF1R promoter indicates demethylation in the tumor samples. Percent cytosine methylation depicted by a scatter plot (1-way ANOVA).

The HELP assay does not distinguish between 5-methylcytosine and 5-hydroxymethylcytosine; therefore, we quantified these using a mass spectrometry approach. We found only around $0.01 \%-0.05 \%$ of DNA to be hydroxymethylated, as compared with the $2 \%-6 \%$ global methylation; therefore, we considered the hydroxymethyl mark to be negligible and not to interfere with our conclusions (Figure $1 \mathrm{H}$ ).

CSF1R is hypomethylated and overexpressed in melanoma in a large subset of samples. Since parallel transcriptomic analysis revealed that immune signaling pathways and cancer-associated pathways were significantly affected by aberrant methylation (Supplemental Table 4), we focused on a common member of both pathways, CSF1R (c-fms), for functional validation. Furthermore, the CSF1R locus was the most significantly demethylated region in locally advanced and metastatic tumors (Figure 1E, blue dot), thus prompting further functional studies. We observed significant positive correlation between mean CSF1R promoter hypomethylation and CSF-1R gene expression (Spearman's correlation, $r=0.41, P=0.011$, Figure 2A) across all samples. In fact, CSF1R overexpression and aberrant promoter hypomethylation was seen in melanomas when compared with melanocyte controls (Figure 2, B and C). The methylation status of $6 \mathrm{CpG}$ sites in the CSF1R promoter region (Figure 3A) was validated at single bp resolution by bisulfite MassARRAY, which demonstrated significant promoter demethylation in malignant melanoma samples (Figure 2D). Invading immune cells are commonly seen in tumor tissue such as melanoma, with macrophages being the most abundant type (14), and CSF-1R is generally highly expressed in macrophages. In order to demonstrate that the CSF1R signal was in fact associated with the tumor cells, we performed IHC, RNA-FISH, and quantitative PCR (qPCR). IHC demonstrated that the CSF1R overexpression seen in the transcriptomic studies was detectable at the protein level, specifically on the MelanA-positive malignant melanoma cells in the original 29 samples, as well as in an additional set of melanomas (Figure 3B and Supplemental Figure 3, A and B), with over $60 \%$ of melanoma samples revealing moderate to high expression (Figure $3 \mathrm{C}$ ). The specificity of the signal was also validated using RNA-FISH on 5 melanoma cell 


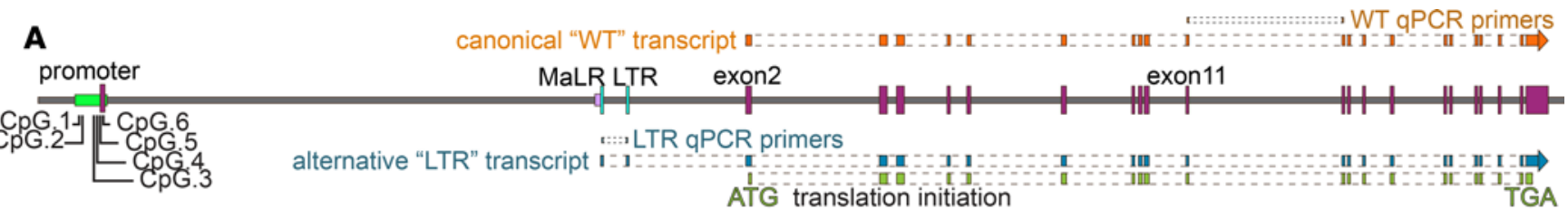

B

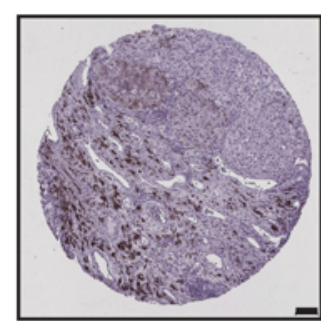

negative

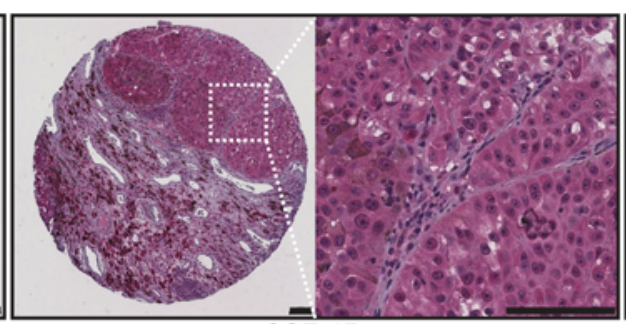

CSF-1R

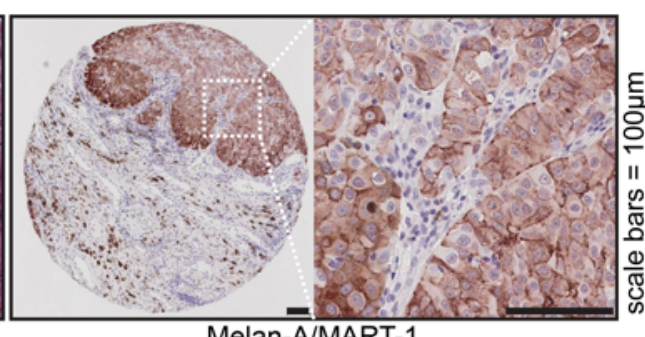

Melan-AIMART-1

C

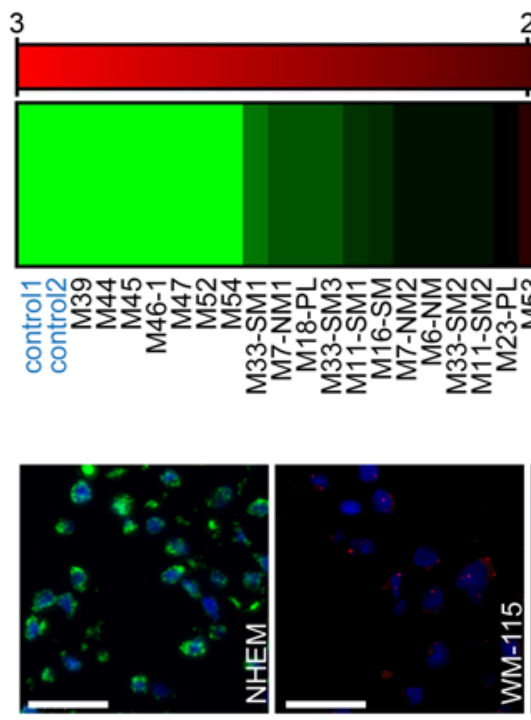

2

1

CSF1R and MLANA
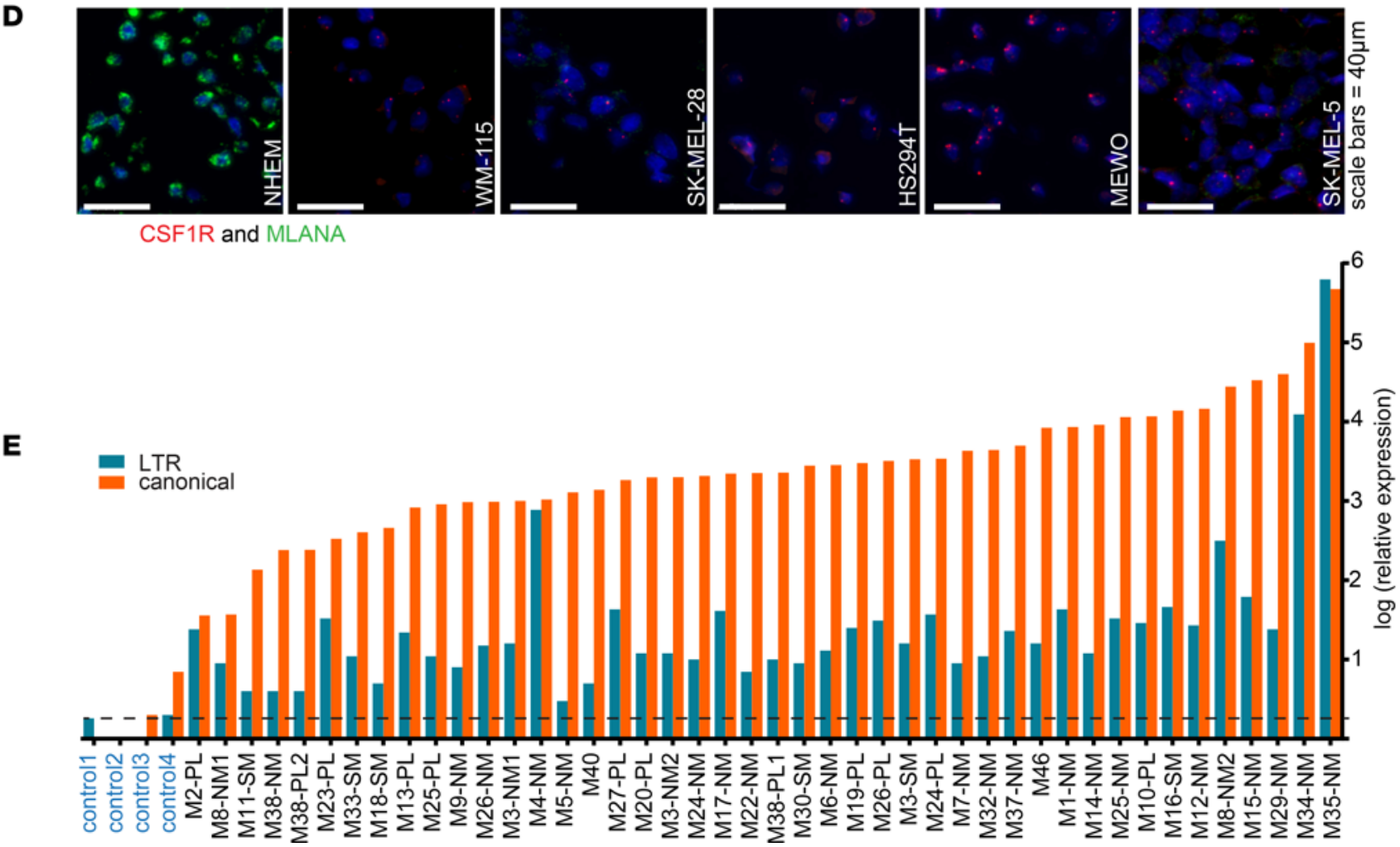

Figure 3. CSF-1R expression in melanoma. (A) The human CSF1R gene is composed of a total of 22 exons (purple rectangles), of which the first exon is noncoding and where the remaining 21 exons (starting with exon 2) encode for the canonical CSF-1R protein. Two additional viral exons (light blue rectangles) are transcribed as part of the LTR transcript. There are 6 differentially methylated CpG sites in the canonical promoter (light green rectangle). Viral sequence (MaLR LTR) denoted with light purple rectangle. (B) Representative images of archival formalin-fixed paraffin-embedded melanoma tissue samples on tissue microarrays shown immunostained for MelanA and for CSF-1R. (C) Staining was compared with a positive control (placen- 
ta). The maximal intensity of VECTOR Red staining was graded on a $0-3$ scale ( 0 , negative; 1 , weak; 2 , medium; 3 , strong staining) and demonstrate expression on the melanoma cells. The controls are labeled in blue. (D) RNA-FISH of CSF1R and MLANA on cells of melanocytic origin: normal human epidermal melanocytes (NHEM) and melanoma cells (WM-155, SK-MEL-28, MEWO, SK-MEL-5, HS294T). (E) qPCR analysis (mean) showing expression of both forms of CSF1R in the melanoma tissue samples. Dashed vertical line showing average LTR expression in controls.

lines (Figure 3D) and revealed CSF1R transcripts in these malignant cells. Furthermore, we also validated CSF1R expression with qPCR on the tumor samples (Figure 3E), as well as 4 additional melanoma cell lines, and we again observed overexpression in melanoma samples (Supplemental Figure 3C).

CSF1R regulates melanoma growth and invasion and is driven by an alternative upstream viral promoter in a subset of cases. In addition to the primary samples, the BRAF V600E mutant cell lines A2058, WM-266-4, and M14c\#5 were studied in an effort to determine the functional relevance of the CSF-1R tyrosine kinase expression in melanoma. Using these and additional cell lines, we assessed the gene expression of CSF1R and related key genes, including the 2 known CSF-1R ligands, CSF-1 and IL-34 (15), and its well-described myeloid regulatory factors PU.1, RUNX1/AML1, and CBFB (16). The CSF1R transcript was detected in all cell lines; however, the myeloid master regulator PU.1 did not show any appreciable expression, signaling the possibility of a different mechanism governing the expression of CSF1R in melanoma. Both subunits of the heterodimeric transcription complex core binding factor (CBF), $C B F \beta$ and the DNA-binding transcription factor RUNX1, were found to be overexpressed in melanoma cells (Supplemental Figure 3C).

A recent report had described the aberrant expression of CSF1R in Hodgkin's lymphoma via an endogenous retroviral element, the long terminal repeat (LTR) of the MaLR THE1B family located $\sim 6.2 \mathrm{~kb}$ upstream of CSF1R coding sequences. This aberrantly initiated form has a different $5^{\prime}$ UTR than the canonical transcript (Figure 3A) but retains the same protein coding sequence (17). We evaluated whether this same mechanism was active in melanoma. qPCR with probes specific for the canonical and aberrant transcripts demonstrated that both were detected in all melanoma samples at higher-than-average levels of controls, with half of melanomas expressing high levels of the aberrant transcript (Figure $3 \mathrm{E}$ ). This was further validated in the 3 BRAF mutant and 1 NRAS mutant melanoma cell line (Supplemental Figure 3C). In contrast, no CSF1R transcript was detected in human epidermal melanocytes according to published RNA-seq data (18) and by our RNA-FISH study (Figure 3D). Furthermore, when evaluating benign melanocytic nevus samples (moles) from healthy donors, we did not detect any LTR form in these cells (Supplemental Figure 3D). In addition to qPCR, the presence of the alternative transcript was further confirmed by sequencing of the $5^{\prime}$ untranslated region unique to this transcript in several of our positive samples (Supplemental Figure 4A). Analysis (19) of the MaLR LTR indicates a putative RUNX1 binding motif (Supplemental Figure 4B), and we therefore decided to focus on RUNX1 as a potential regulator of CSF1R expression in melanoma.

Stable cell lines harboring knockdowns of CSF1R and RUNX1 in V600E mutant melanoma cells were created (Figure 4, A and B). shRNA mediated knockdown of CSF1R and RUNX1 mRNAs resulted in significantly slower growth and longer doubling times in 2-D cell culture (Figure 4, C and D, and Supplemental Figure 5A) and significantly smaller colonies in the 3-D Matrigel culture (Figure 4E and Supplemental Figure 5B). Additionally, we utilized a specific small-molecule inhibitor of CSF-1R kinase PLX3397 (20, 21) for all functional studies. Pharmacological inhibition of CSF-1R also resulted in smaller colonies in 3-D culture (Figure 4F) due to lower proliferative and higher apoptotic rates (Figure 4G and Supplemental Figure 5C). The invasiveness of melanoma cells also showed a dose-dependent decrease after treatment with PLX3397 (Figure 4H and Supplemental Figure 5D). The stable CSF1R knockdowns also showed decreased invasion (Figure 4I), demonstrating a functional role for the CSF-1R in the growth and spread of melanoma.

Expression of the CSF-1R and related proteins increases during the rebound of ERK activation after BRAF inhibition. We queried the Cancer Genome Atlas (TCGA) melanoma biospecimen database generated by the TCGA Research Network (http://cancergenome.nih.gov/) to determine the associations between CSF1R promoter methylation and gene expression in an independent large cohort $(n=471)$ and to analyze its association with particular mutational subgroups. CSF1R methylation (probe cg23505299) was strongly inversely correlated with expression (Spearman's correlation, $r=-0.3995, P<0.0001$, Figure 5A). Across all TCGA melanoma samples, we also observed a positive correlation between the expression of CSF1R with the transcription factor RUNX1 (Spearman's correlation, $r=0.4637, P<0.0001$ ) (Figure 5B) or with its ligand, IL34 ( $r=0.5334, P<0.0001)$ (Figure 5C) (2). Furthermore, melanomas with activating mutations in the canonical MAPK pathway (BRAF, NRAS, or NF1 driver mutations) displayed significantly lower 

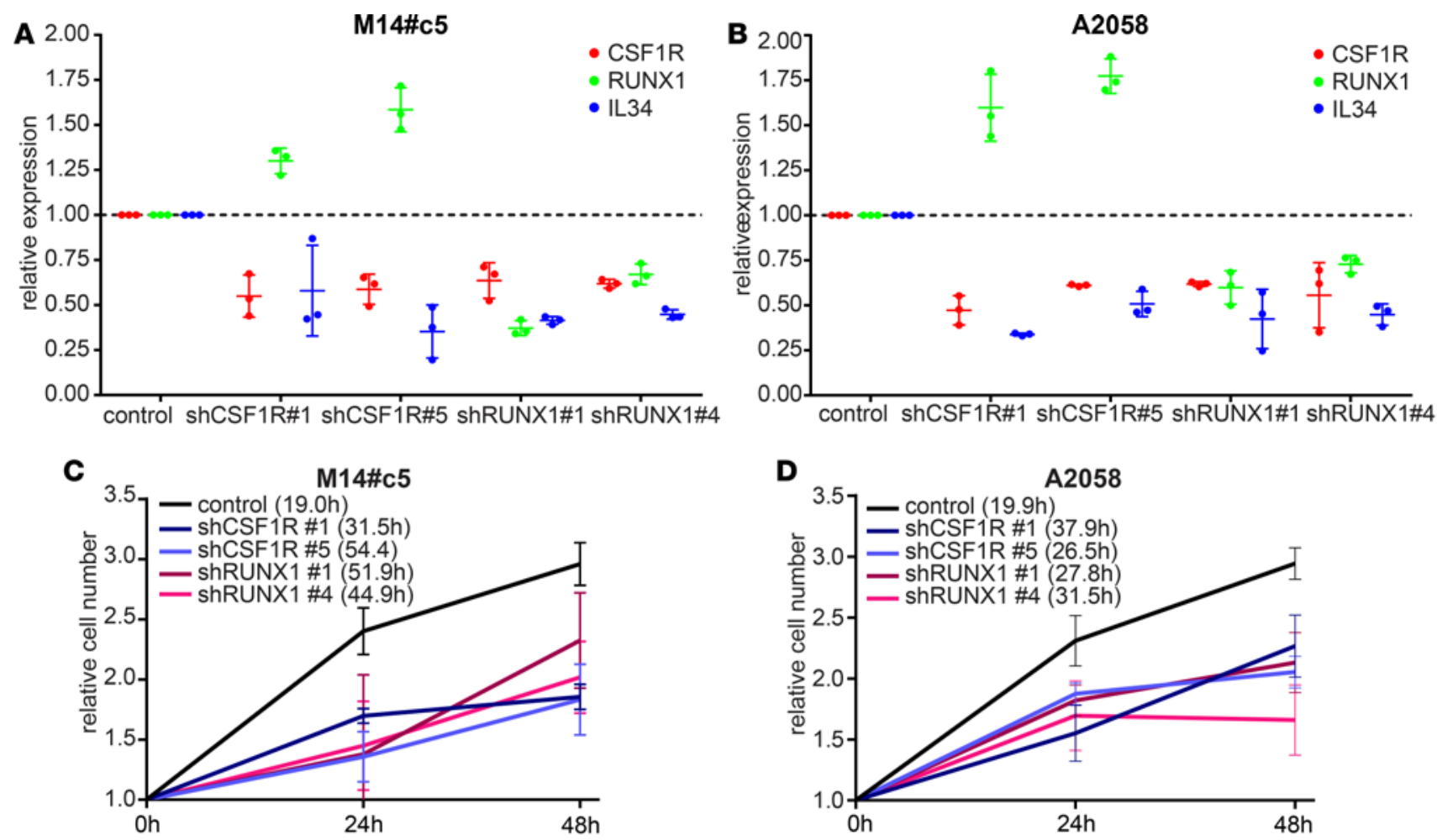

E

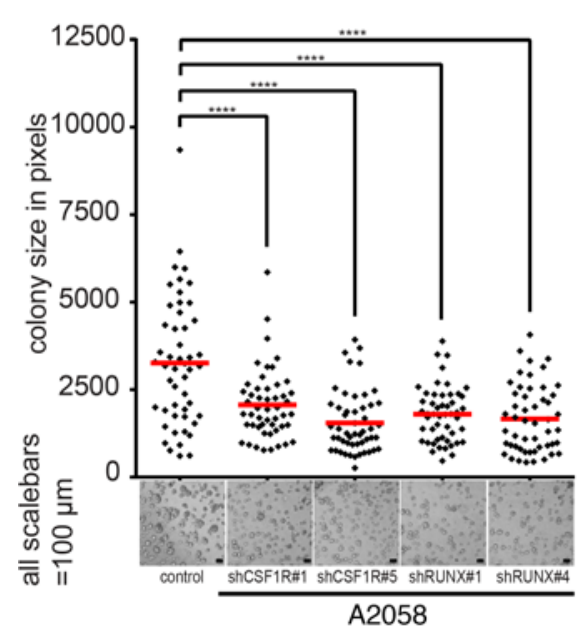

$\mathbf{F}$

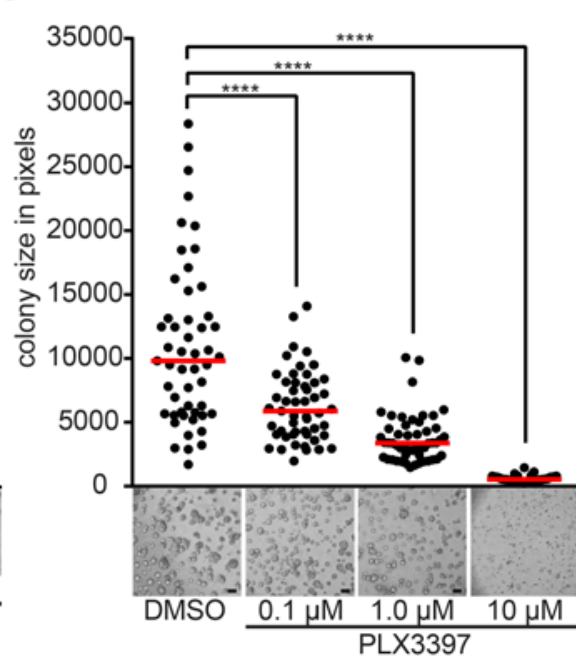

G

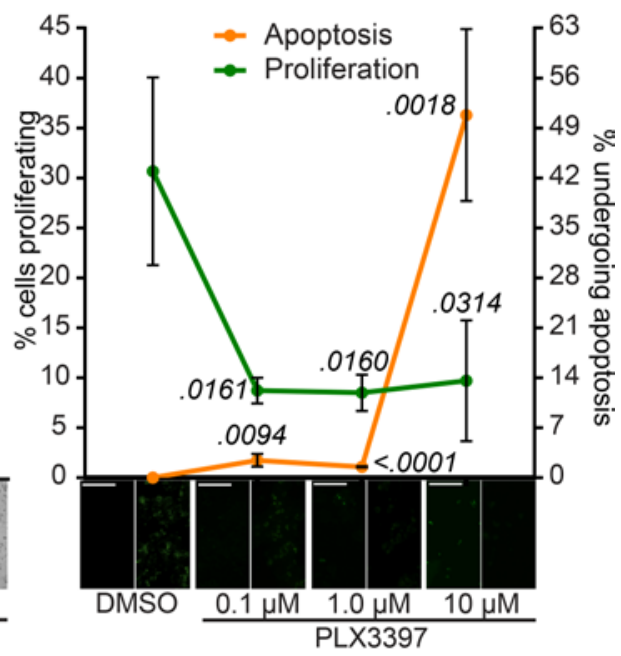

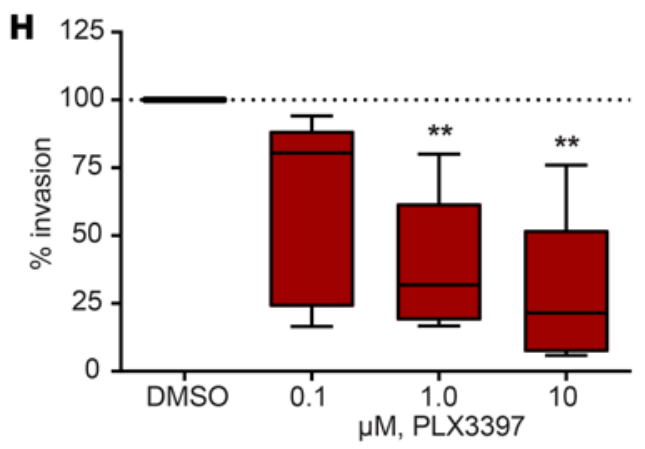

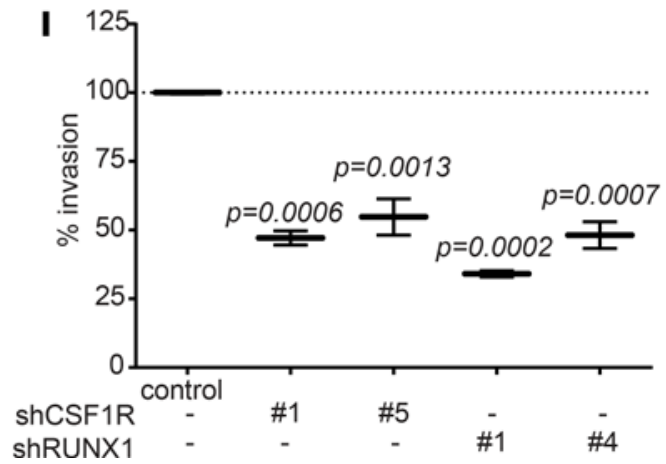


Figure 4. CSF1R expression is activated by an aberrant upstream promoter, and the CSF-1R regulates melanoma growth and invasion. (A and B) Five different lentiviral shRNAs were evaluated for CSF1R and RUNX1. Among these, shRUNX1\#1, shRUNX1\#4, shCSF1R\#1, and shCSF1R\#5 conferred the strongest knockdown. Therefore, these were used for all subsequent experiments. Gene-expression analysis of CSF1R, RUNX1, and IL-34 in melanoma cell lines M14c\#5 (A) and A2058 (B) stably harboring shRNA species against CSF1R or RUNX1 (3 biological replicates). (C-F) Proliferation of the stable knockdown cell lines with the doubling times (hours) in parentheses (C and $\mathbf{D}$ ); for statistical analysis see Supplemental Figure 5A. Three-dimensional Matrigel cell culture of the stable A2058 cells (E) and the parental A2058 cells cultured with increasing dose of PLX3397 (F). (G) Pictures taken on the fifth day of culture; red line, median colony size in pixels. Quantitative analysis of cells undergoing proliferation and apoptosis in the 3-D cultures ( $t$ test, 2-tailed). ( $\mathbf{H}$ and $\mathbf{~ I ) ~ L a r g e r ~ i m a g e s ~ o f ~ a l l ~ 3 - D ~ c u l t u r e s ~ a r e ~ p r o v i d e d ~ i n ~ S u p p l e m e n t a l ~ F i g u r e ~ 5 , ~ C ~ a n d ~ D ~ ( r e p r e s e n t a t i v e ~ i m a g e s , ~ 3 - D ~ e x p e r i m e n t s ~}$ performed twice). Transwell invasion assay (mean \pm SEM) of A2058 cells using the CSF-1R inhibitor PLX3397 (H) and of A2058 stable RUNX1 or CSF1R knockdowns (I); images represent 3 biological replicates. All statistical analyses of 3-D colony size and invasion assays: 1-way ANOVA (statistical significance levels are noted with asterisks, $\left.{ }^{* *} P \leq 0.01,{ }^{* * *} P \leq 0.0001\right)$.

CSF1R methylation (two-tailed $t$ test, $P=0.001$ ) (Figure 5D) and significantly higher CSF1R expression compared with the triple-WT specimens (two-tailed $t$ test, $P=0.04$ ) (Figure $5 \mathrm{E}$ ). Thus, we next examined the functional significance of CSF-1R in ERK signaling in melanomas.

The MAPK/ERK pathway is hyperactivated in a high percentage of melanoma tumors due to activating mutations in BRAF and other members of the pathway. Treatment with V600 RAF inhibitors potently inhibit the mutant protein, resulting in rapid inactivation of the MAPK pathway. However, this initial inhibition of ERK activity is quickly followed by a rebound in ERK phosphorylation, displaying restored MAPK activity due to activation of tyrosine kinases such as PDGF and EGFR activated by derepressed ligands due to loss of constitutive BRAF activation (22-26). Since, CSF-1R ligand engagement in myeloid cells has been shown to activate the PI3K/AKT and the ERK signaling pathways (27-29), we determined the role of CSF-1R on the rebound of ERK signaling after BRAF V600E inhibitor treatment. Melanoma cells with BRAF V600E mutation were treated with the specific V600E inhibitor PLX4720 $(30,31)$ for up to 96 hours. This resulted in a significant decrease in viability, as expected. During the emergence of a BRAF inhibitor-resistant cell population (23), an increase in the $130-\mathrm{kDa}$ CSF-1R precursor protein, and the appearance of the higher-molecular weight $(165 \mathrm{kDa})$, mature version of CSF-1R that may be expressed on the cell surface was observed. We also detected an increase of the surface expression of CSF-1R via flow cytometry after a 96-hour exposure to the BRAF inhibitor (Figure 5, F and G). Transcriptionally, we observed the expression of RUNX1 and both forms of CSF1R mRNA increase with treatment duration, peaking on or after 72 hours (Figure $5, \mathrm{H}$ and I). The mRNA of the CSF-1R ligand IL-34 was significantly elevated, as was the level of secreted IL-34 protein rising, along with the duration of BRAF inhibitor treatment (Figure 5, J and K). Stable melanoma cell lines, harboring a luciferase reporter linked to either the full canonical promoter of CSF1R or the viral LTR sequence, were generated. We observed that BRAF inhibitor treatment was associated with an increase in activity at both the canonical and alternative promoter with time, indicating that the viral LTR functions as a bona fide promoter in melanoma cells (Figure 5, $\mathrm{L}$ and $\mathrm{M}$ ).

The CSF1R is overexpressed in melanoma samples resistant to inhibitors of the MAPK pathway. In order to evaluate the levels of CSF-1R, RUNX1, and IL-34 in V600E mutant melanoma patients with refractory disease after RAF and/or MEK inhibitor treatment, we analyzed 3 recently published clinical datasets from the following cohorts: (a) A cohort of 6 patients (Van Allen et al.; ref. 32) who developed resistance to either single-agent RAF inhibition (1 patient: vemurafenib) or combined RAF and MEK treatment (5 patients: dabrafenib and trametinib); (b) a cohort of another 6 patients (Sun et al.; ref. 23), including 5 patients treated with a RAF-inhibitor (dabrafenib or vemurafenib) and 1 patient who relapsed after trametinib treatment; and (c) a cohort of 13 patients (Kwong et al.; ref. 33), 3 of whom were on vemurafenib and an additional 10 of whom were on dabrafenib plus trametinib treatment. For all patients, whole transcriptome sequencing data were analyzed from paired melanoma biopsies taken before and during treatment (when available), as well as after relapse. We observed upregulation of CSF1R, IL34, and RUNX1 in resistant samples compared with the matched pretreatment tumors (Figure 6A). In 2 of the RAF inhibitor-resistant tumors, 2 of the RAF/MEK inhibitor-resistant samples, and the 1 patient with MEK inhibitor resistance, all 3 genes were overexpressed. With the exception of 1 patient, CSF1R mRNA levels were always elevated in the resistant samples, with either IL-34 mRNA, RUNX1 mRNA, or both upregulated as well. Compared with the pretreatment samples, the upregulation of CSF1R mRNA was highly significant (median expression 5-fold higher) in the resistant biopsies (Figure 6B).

Coinhibitory treatment of BRAF V600E and CSF-1R is synergistic in melanoma. After observing an increased expression of CSF-1R, RUNX1, and IL-34 coinciding with the rebound from BRAF inhibition and with the development of resistance in patients, we determined the efficacy of combination therapy with PLX3397 and 

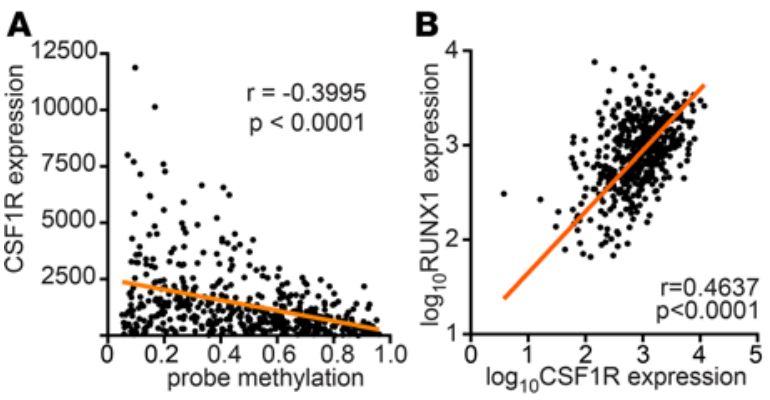

$\mathbf{F}$

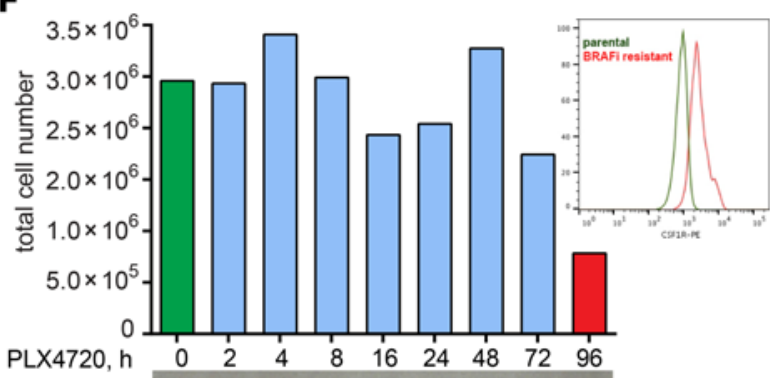

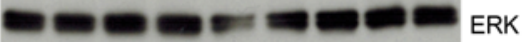

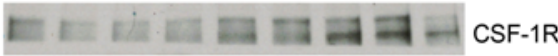

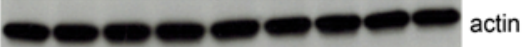
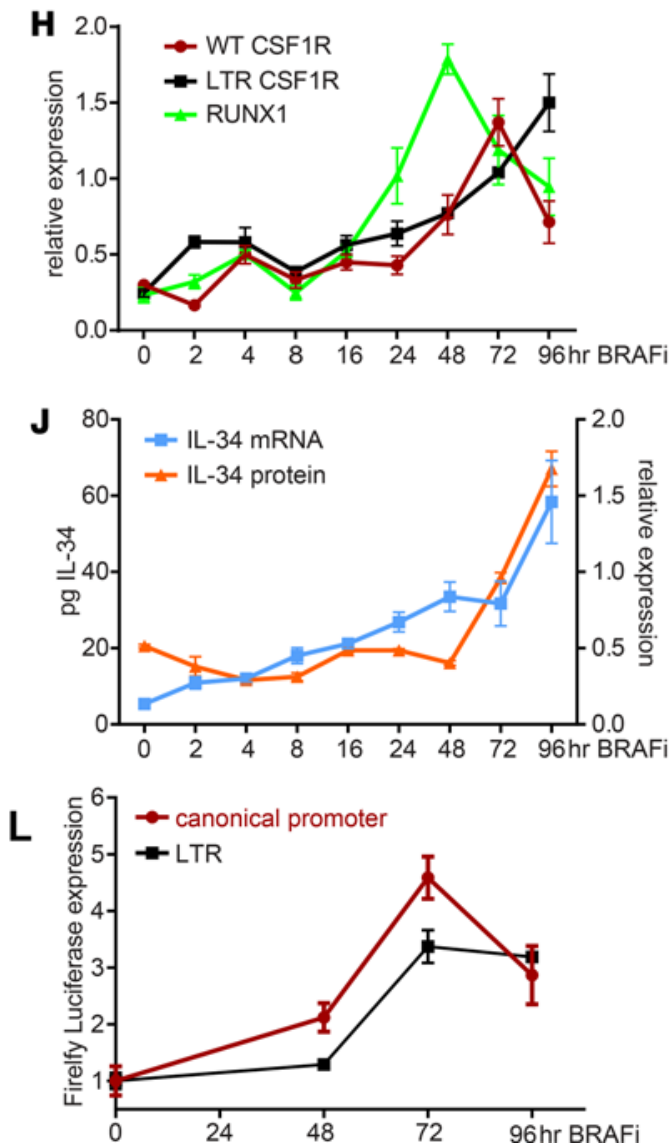
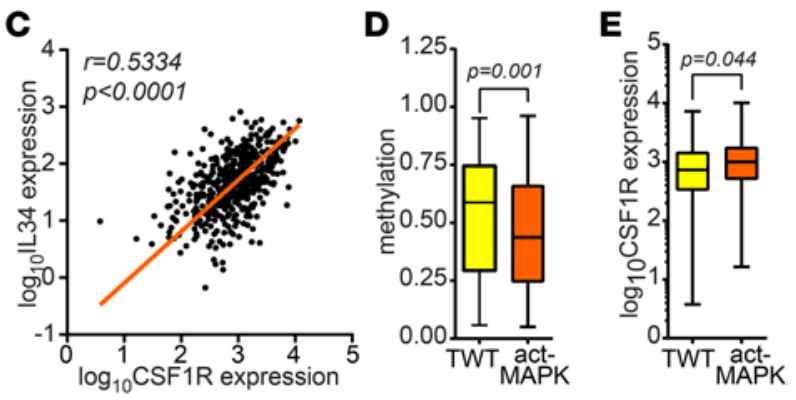

G
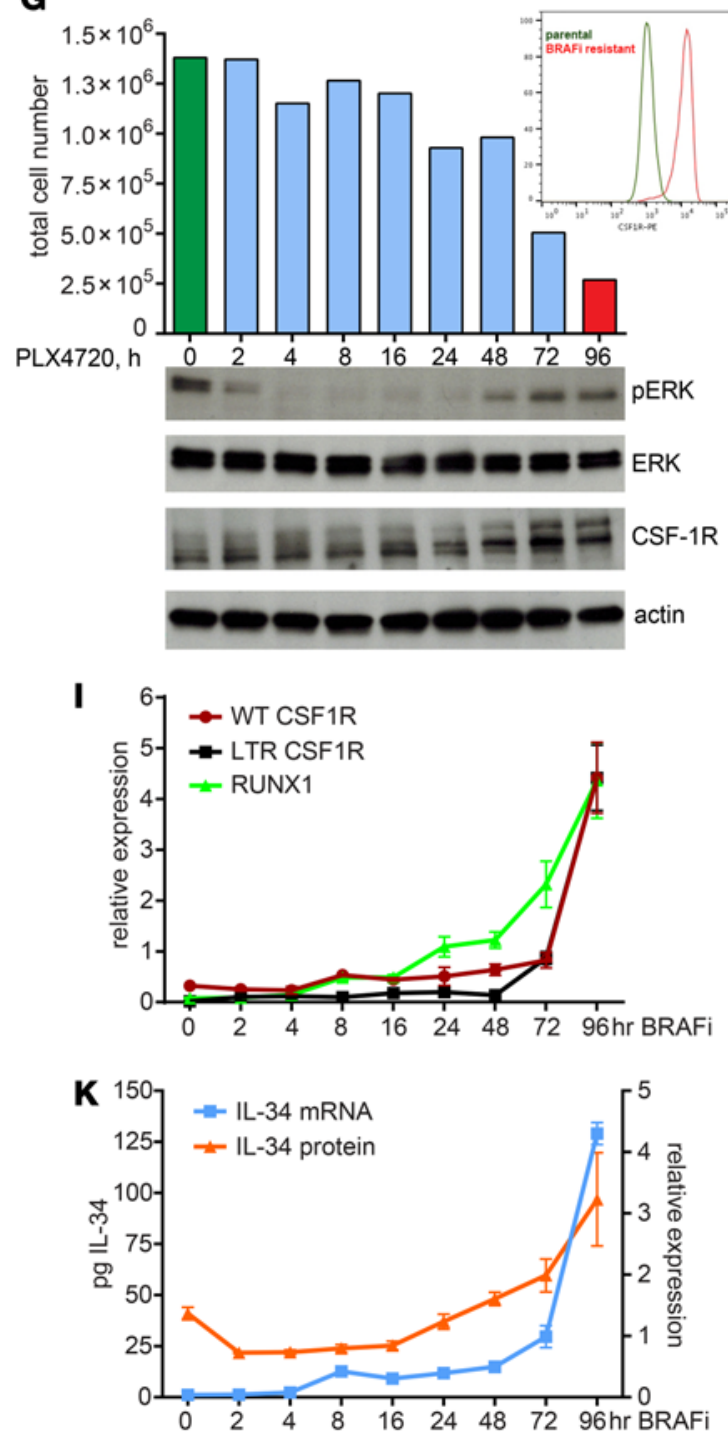

M

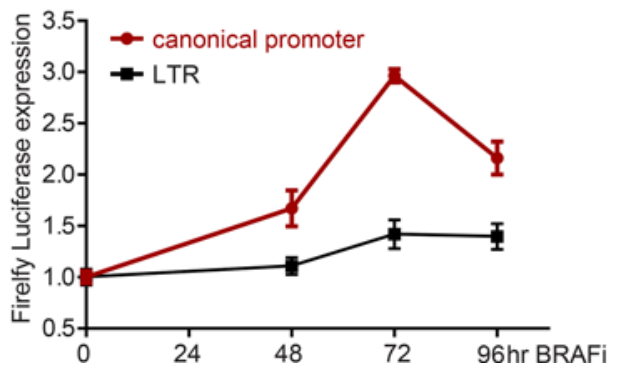


Figure 5. ERK-pathway rebound following 96-hour BRAF V600E inhibitor treatment is associated with increased expression of RUNX1, CSF1R, and IL34. (A) CSF1R probe methylation shows negative correlation with CSF1R gene expression across all 471 TCGA melanoma samples (Spearman's correlation, $r=-0.3995, P<0.0001$, orange line denotes linear regression). (B and C) CSF1R expression shows positive correlation with that of $R U N X 1$ ( $r=0.464, P<$ $0.0001)$ (B) or with the expression of $I L 34(r=0.533, P<0.0001)$ (C). ( $\mathbf{D}$ and $\mathbf{E})$ CSF1R probe methylation (D) and gene expression (E) of TCCA melanomas with MAPK-activating mutation (act-MAPK) and without (triple WT, TWT) (unpaired $t$ test, 2-tailed). (F-M) A2058 (F, H, J, L) or WM-266-4 (G, I, K, M) cells were treated with $3 \mu \mathrm{M}$ PLX4720 and/or serum-free medium for a total of 96 hours. (F and $\mathbf{G}$ ) Cell lysates assayed for gene expression and protein expression; supernatants assayed for secreted proteins. Detailed schematic of the experiment is provided in Supplemental Figure 7A. Melanoma cell numbers were counted for every time-point condition. Immunoblotting of total protein extracts from all time points was performed to detect protein levels of phospho-ERK, total ERK, and actin, as well as to evaluate both the levels and posttranslational maturation of CSF-1R protein. Surface expression of CSF-1R protein was followed by flow cytometry (small inserts). (H and I) qPCR analysis (mean \pm SEM) for relative expression of RUNX1 and for both transcripts of CSF1R. (J and K) qPCR of IL-34 expression and ELISA analysis of IL-34-secreted protein expression (mean \pm SEM for both). (L and $\mathbf{M}$ ) Luciferase activity in melanoma cells stably harboring a plasmid construct of the canonical promoter of CSF1R or the CSF1R MaLR LTR linked to Firefly luciferase show increased activity at both regulatory regions (mean \pm SEM). Representative images from 3 independent experiments.

PLX4720 in traditional cell culture, in 3-D cell culture, and in a murine xenograft model of the A2058 cell line. First, we tested the inhibitory effect of both drugs on melanoma cell proliferation alone and in combination, and we calculated the combination indices $(\mathrm{CI})$ at half maximal effective concentration $\left(\mathrm{EC}_{50}\right)$, as well as at $\mathrm{EC}_{40}$ and $\mathrm{EC}_{75}$ (34). We found the combined effect to be synergistic in multiple melanoma cell lines and comparable with the therapeutic effect seen in the combination of trametinib and PLX4720 in vitro (Figure 7A and Supplemental Figure 6, A-C). Next, growing 3-D melanoma cultures were treated with the 2 drugs in several different concentration combinations, leading to a significant inhibitory effect even when using lower concentrations for both drugs (Figure 7B and Supplemental Figure 6, D-F). In vivo efficacy of the combination was also tested in a murine xenograft model. Treatment of A2058 melanoma xenografts with the CSF$1 \mathrm{R}$ inhibitor $(P=0.0003, \log$ rank test $)$ or the BRAF inhibitor $(P=0.0012)$ individually increased survival in mice. However, combination of both inhibitors led to even more significantly increased survival $(P<0.001)$ demonstrating the potential efficacy of this strategy (Figure 7C and Supplemental Figure 6G).

Finally, in an effort to analyze the effect of CSF-1R inhibition on ERK pathway reactivation, we added PLX3397 in the last hour of the PLX4720 time course experiment to each time point (Supplemental Figure 7A). Without the CSF-1R inhibitor, we saw the ERK phosphorylation signal reappearing around 48 hours of continuous V600E inhibition and, by hour 72, the rebound was complete. The levels of phospho-AKT also slowly increased during the rebound, indicating association of the PI3K/AKT pathway during acquisition of resistance. The addition of PLX3397 resulted in the abrogation of ERK activation, as well as AKT phosphorylation, indicating that these pathways are driven downstream of this receptor in melanoma (Figure 7D and Supplemental Figure 7B). Furthermore, using recombinant IL-34 or CSF-1, we were able to reactivate ERK-phosphorylation after 1-2 hours of BRAF inhibition and observed increased AKT phosphorylation. Both ERK-phosphorylation and AKT phosphorylation were attenuated by PLX3397 being coadministered with either ligand (Figure 7E and Supplemental Figure 7C).

\section{Discussion}

During the past decade, our understanding of melanoma has progressed rapidly, leading to an improvement in overall survival for patients with advanced disease. Those with BRAF V600E mutant melanoma have seen improvement in median overall survival from well under 12 months (6-9 months) to over 2 years. However, the majority of patients treated with the best MAP kinase targeted therapy (BRAFi + MEKi) still relapse by around 1 year, and nearly all will ultimately result in relapse of the disease. Despite the novel therapeutics and the advances, there is still a pressing need for an even better understanding of the genetic and epigenetic mechanisms that lie behind melanoma development and progression, as well as confer resistance to these targeted therapies. Therefore, we took an integrated epigenomic approach to look for novel therapeutic targets in melanoma to potentially combat resistance to the currently accepted modalities. The key findings of our study include the following: (a) the melanoma genome is characterized by widespread demethylation; (b) CSFIR is aberrantly demethylated and is expressed via an endogenous viral promoter in a subset of melanomas, and (c) RUNX1, CSF1R, and IL34 overexpression correlates with progression, invasion, and acquired resistance to inhibitors of the MAPK pathway.

After conducting a genome-wide analysis of cytosine methylation in melanoma, we demonstrate that loss of methylation, rather than hypermethylation, is the more pervasive global epigenetic alteration that is seen in this disease, in line with other reports (35). Furthermore, our data indicate that demethylation occurs early in melanomagenesis and can be observed in both early and advanced tumors. CSF1R was associated with 
A
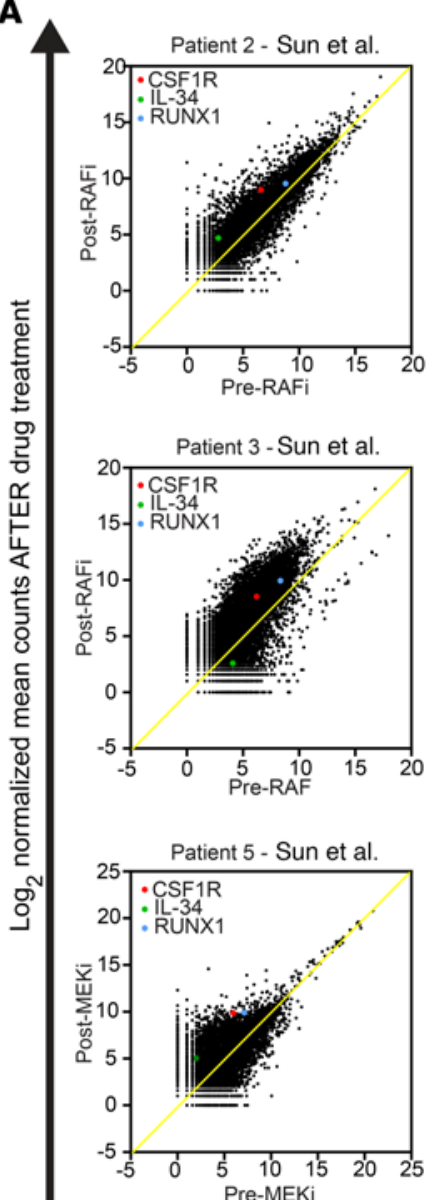
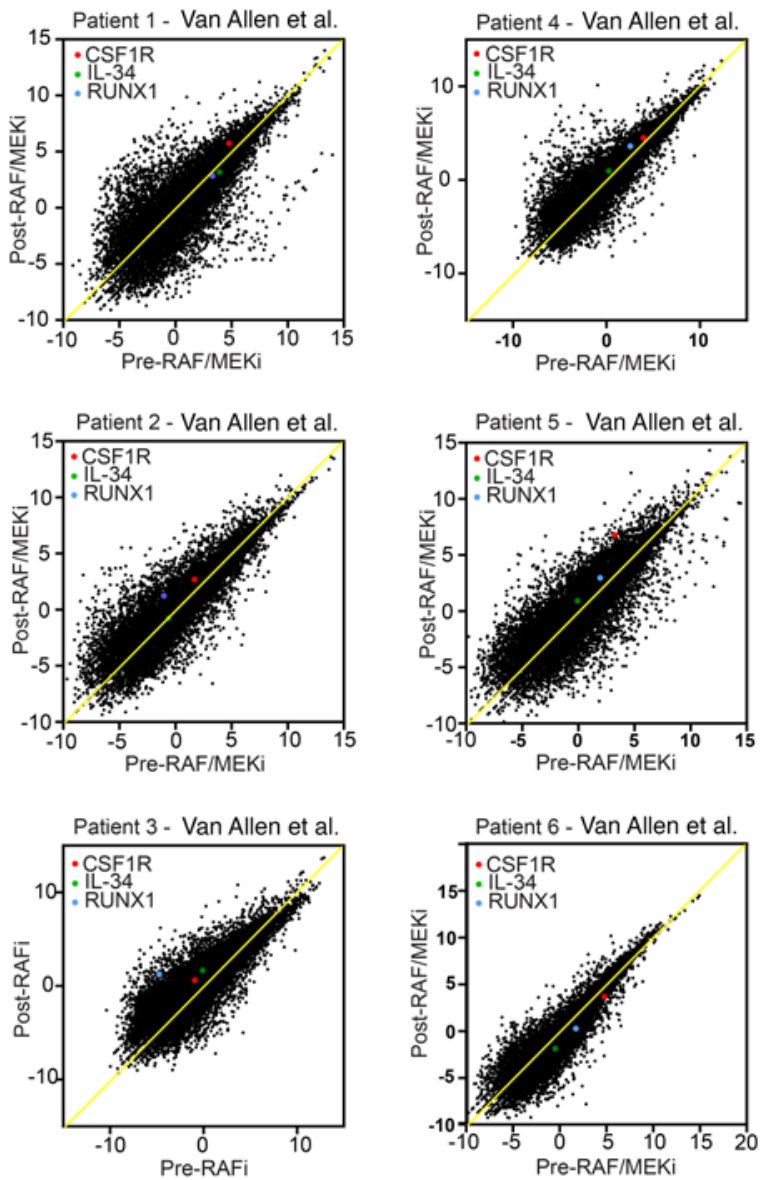

Figure 6. Gene expression analysis of CSF1R, RUNX1, and IL-34 in refractory BRAF V600E melanoma patients. (A)

Scatter plots of $\log _{2}$ normalized read counts between pre- and posttreatment (resistant tumors) with RAF, MEK, or RAF/MEK inhibition in 9 BRAF V600E melanoma patients. (B) Changes in expression of CSF1R, IL-34, and $R U N X 1$ while on treatment of MAPK pathway inhibitors (On-TX) or in refractory tumor samples compared with the pretreatment biopsies (Pre-TX) (1-way ANOVA)

$\log _{2}$ normalized mean counts BEFORE drug treatment

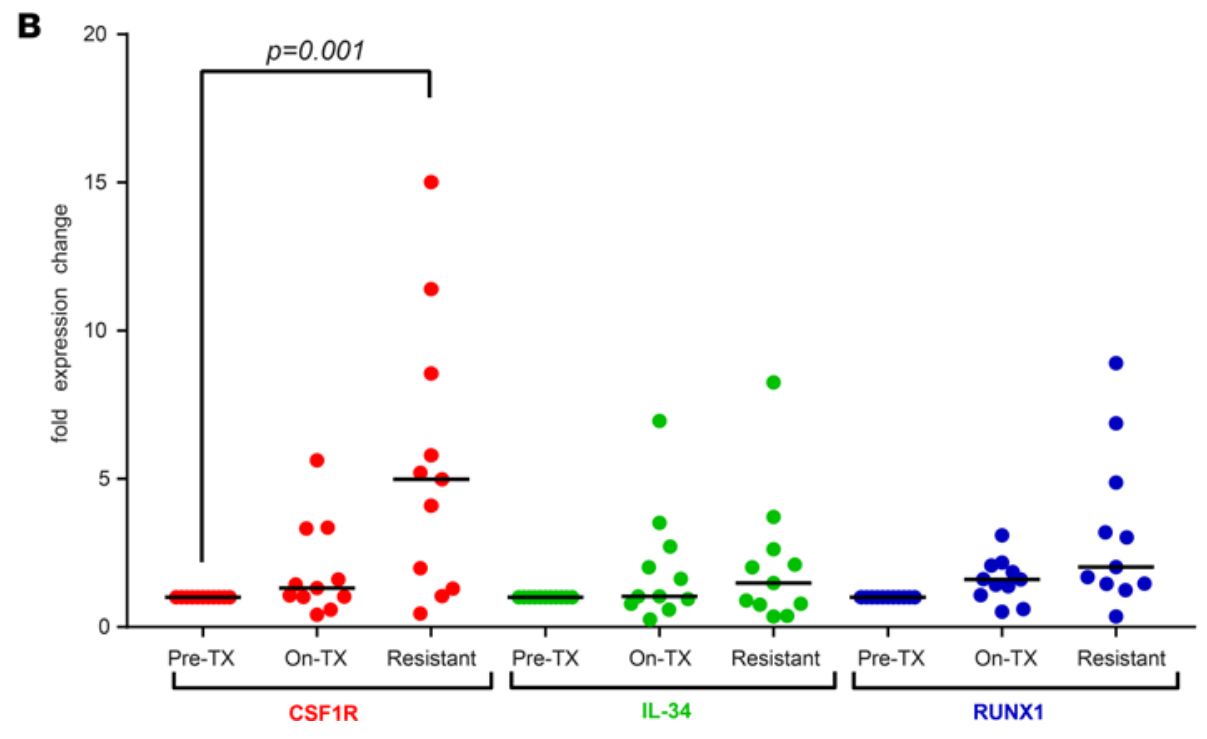

promoter hypomethylation and overexpression in our melanoma tumors, as well as in the TCGA samples. Additionally, it was the most significantly demethylated gene in locally advanced and metastatic tumors.

CSF-1R is expressed by cells of the myeloid-lineage and is a marker of macrophage differentiation (29). The tumor microenvironment consists of various cell types, in addition to the malignant cells; the most abundant at every stage are the innate leukocytes, notably the tumor associated macrophages (TAMs) read- 
A

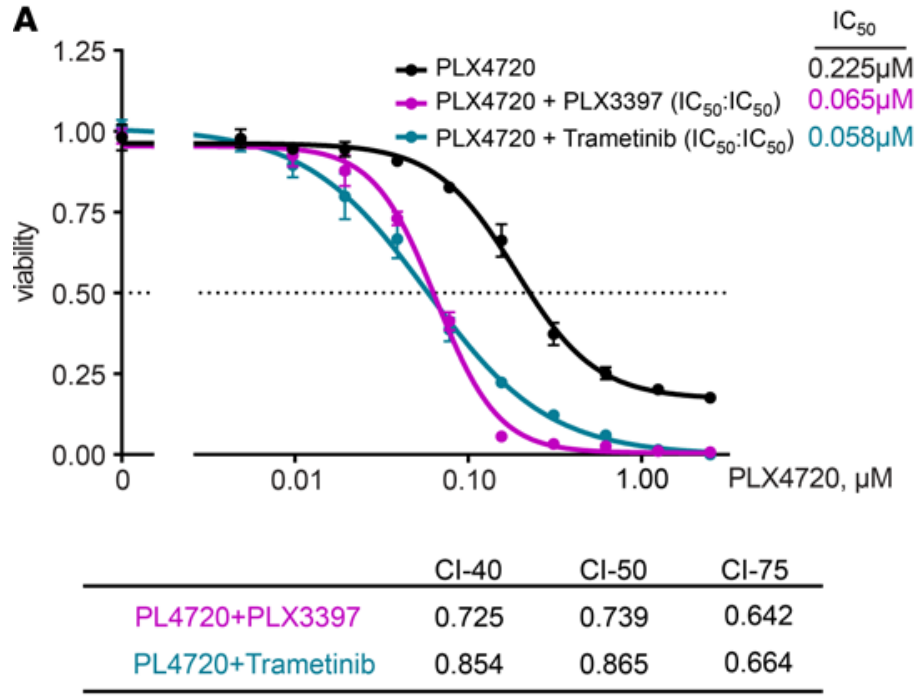

B

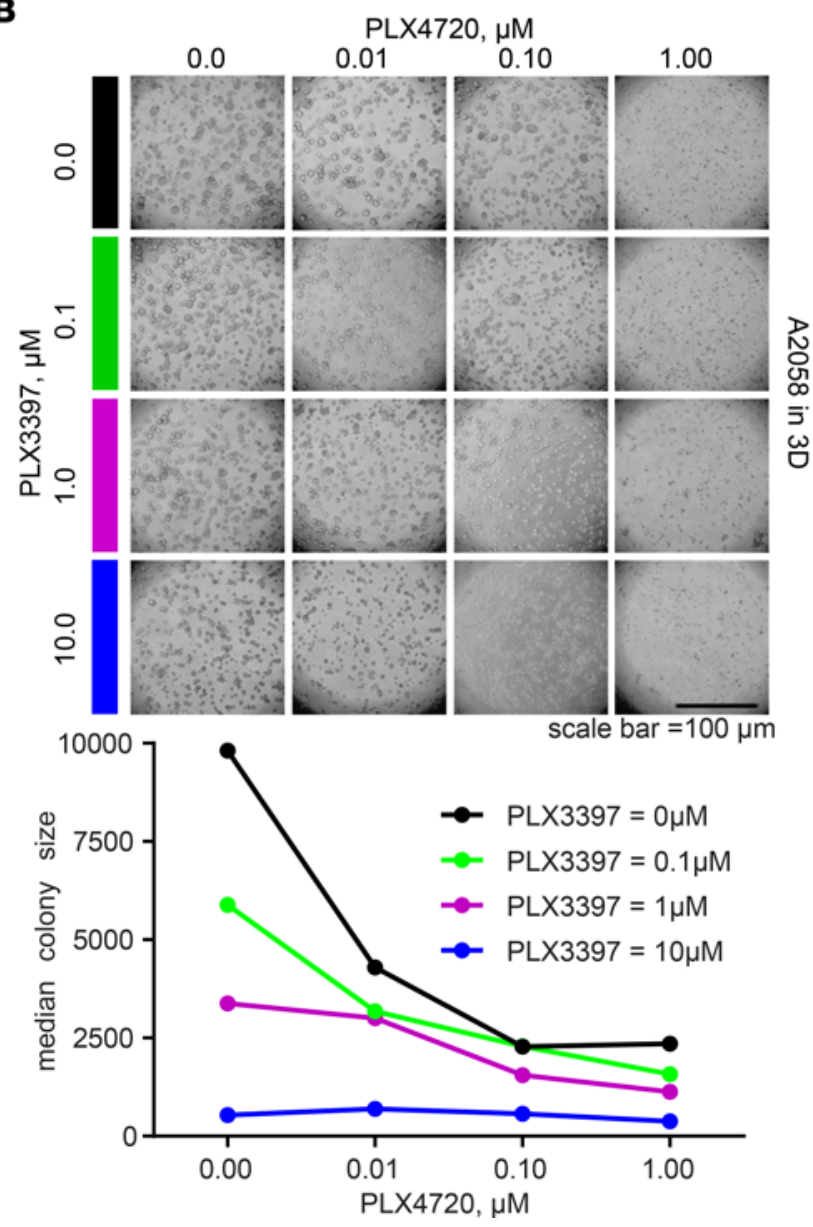

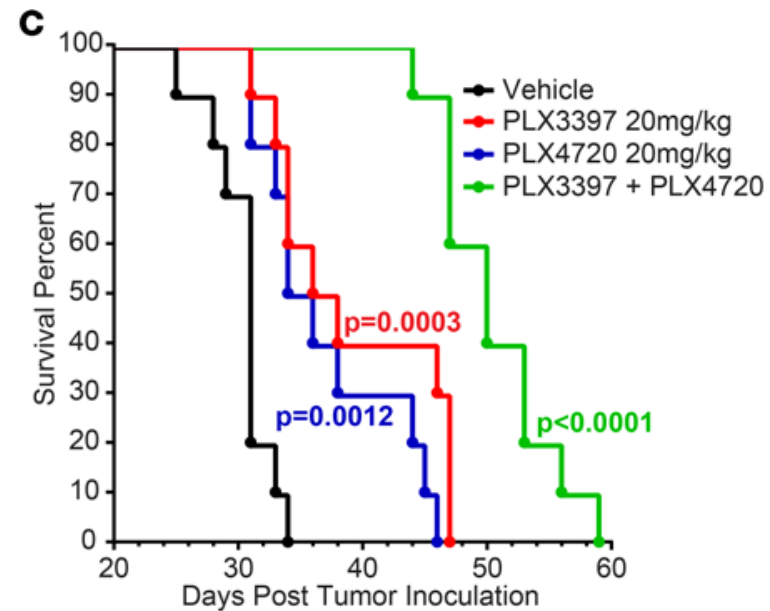

D

$\begin{array}{llllllllll}P L X 4720 & 0 & 2 & 4 & 8 & 16 & 24 & 48 & 72 & 96 h\end{array}$ (no PLX3397)

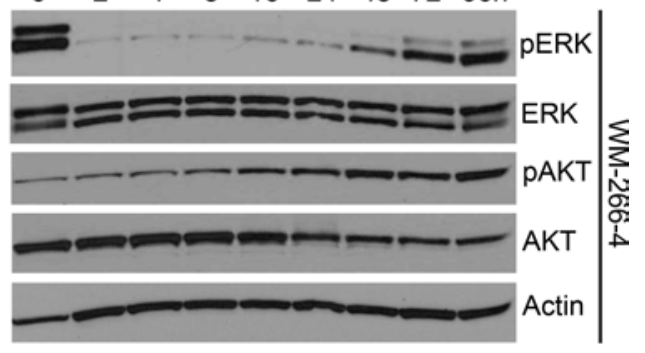

$\begin{array}{llllllllll}P L X 4720 & 0 & 2 & 4 & 8 & 16 & 24 & 48 & 72 & 96 \mathrm{~h}\end{array}$ (1h PLX3397)

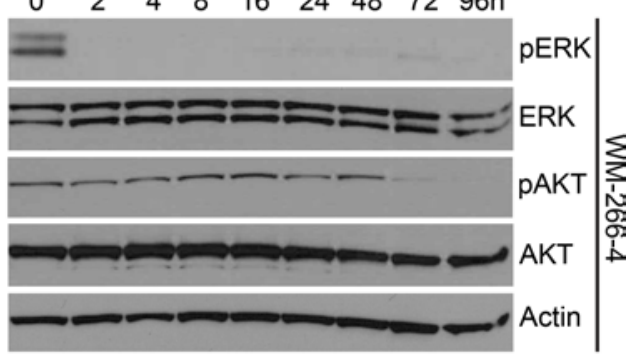

$\mathbf{E}$

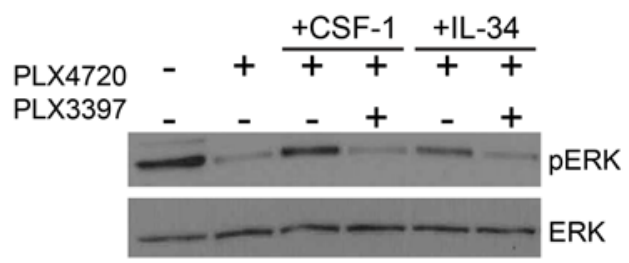

Figure 7. Coinhibition of BRAF V600E and CSF-1R is synergistic in melanoma. (A) Using a constant ratio, the V600E inhibitor was combined with either the CSF-1R inhibitor or a MEK inhibitor based on the $\mathrm{IC}_{50}$ value of each drug and used to treat melanoma cells in quadruplicate for 72 hours. The alamarBlue assay was then performed to estimate cell growth inhibition, and curves (mean \pm SEM) are shown with the combination index (CI) indicated in the table below for each condition where values <1 demonstrate synergy. (B) Micrographs taken on the fifth day of A2058 3-D cell culture grown with serial dilutions of PLX3397 or PLX4720, as well as of the combination of these 2 inhibitors. Growth inhibition effect of these conditions depicted as median colony size shown. Fifty colonies were measured for each condition. For statistical analysis, see Supplemental Figure 6, D, E, and F. (C) Kaplan-Meier survival curve of A2058 xenografted mice (10 in each group) treated with PLX3397 or PLX4720 or with PLX3397 plus PLX4720 demonstrating significant improvement with combination (log-rank [Mantel-Cox] test, $P$ values indicated in Supplemental Figure 6C). (D) The effect of combined PLX3397/PLX4720 treatment on signaling in the WM-266-4 cell line as depicted by immunoblotting against phospho-ERK, total ERK, phospho-AKT, total AKT, and actin. Cells were lysed after a 96-hour, $3 \mu \mathrm{M}$ PLX4720 time-course treatment with or without 
$30 \mu \mathrm{M}$ PLX3397 added in the last hour. (E) The effect of IL-34 and CSF-1 on the rebound of ERK phosphorylation in A2058 cells as demonstrated by immunoblotting detecting phospho-ERK1/2, total ERK1/2, and actin. Total melanoma cell extract were probed after a 2-hour treatment of $3 \mu \mathrm{M}$ PLX4720 with or without $30 \mu \mathrm{M}$ PLX3397, with $100 \mathrm{ng} / \mathrm{ml}$ rCSF-1 or rlL-34 added in the last 15 minutes.

ily expressing the CSF-1R. There have been numerous studies where the tumor cells themselves have been found to express CSF-1, and in a few cases, coexpression of the receptor has been shown, as well. Among these cancers are the malignancies of the female reproductive system, renal cell carcinoma, and prostate and breast cancer where expression of CSF-1R or CSF-1 can predict poor prognosis. Previous studies have shown that inhibition of macrophage CSF-1R can be potentially efficacious in breast and thyroid cancers (36-39). Some studies reporting efficacy of CSF-1R inhibition in melanoma used the B16 mouse melanoma cell line, which does not express the murine Csflr or Il34 at all. These studies therefore hypothesized that CSF-1R targeting inhibits TAMs only (40-44). Our study is the first to our knowledge to report the aberrant expression of this receptor tyrosine kinase in the melanoma cells themselves, coexpressed with its ligand IL-34, along with an evaluation of its transcriptional regulation and of its potential role in melanoma growth, invasion, and resistance to inhibitors of the MAPK pathway currently used in the clinic.

CSF1R has been reported to be oncogenic in Hodgkin's lymphoma activated by an alternative viral promoter, the MaLR LTR of the THE1B family located upstream of the transcription start site (17). Our data show that aberrant transcription of CSF1R driven by the same LTR sequence is also seen in a subset of melanoma tumors and in all of our BRAF and NRAS mutant cell lines. This finding provides additional support for endogenous viral sequences functioning as promoters contributing to oncogene activation in cancer.

CSF-1R expression in the myeloid lineage is largely dependent on the activity of the transcription factors PU.1 and RUNX1, but the former was undetectable in any of our cell lines. This led us to believe that, in melanoma, a distinct molecular mechanism is primarily responsible for the transcriptional regulation of CSF1R. The canonical promoters of CSF1R and its ligand IL34 (Supplemental Figure 4, C-F) are rich in putative RUNX1/AML-1 binding sites, and analysis of the MaLR LTR also revealed a RUNX1 binding site. Our RNAi studies show that the knockdown of RUNX1 results in lower expression of both CSF-1R and IL-34. At the same time, melanoma cells harboring shCSF1R display higher-than-baseline RUNX1 expression, pointing to a potential compensatory feedback mechanism. Therefore, we propose that, in melanoma, the main regulator of the IL34/CSF1R axis might be RUNX1. Interestingly, we also observed lower-than-baseline expression of $I L 34$ in the shCSF1R-stable cells, signaling the possibility of coordinated expression between the receptor and its ligand involved in the autocrine loop - potentially similar to CSF$1 \mathrm{R}$ and the CSF1 ligand in rat myogenic cells (45).

We investigated the functional role of CSF1R in melanoma utilizing cell lines in traditional 2-D and in 3-D Matrigel cell culture, as well as mouse xenografts. We show that both an RNAi-based loss-of-function approach and the small molecule specific inhibitor PLX3397 attenuates proliferation and invasion both in vitro and in vivo. The feedback activation of receptor tyrosine kinases, such as PDGFR $\beta$, IGF-1R, HGF, MET, and EGFR, have been described to contribute to BRAF inhibition resistance by either increasing RAS activity or through activating parallel pro-proliferative signaling pathways in melanoma $(23-25,46)$.

CSF1R was also found to be hypomethylated and overexpressed in untreated TCGA melanoma patient samples with an activated ERK pathway driven by mutations in BRAF, NRAS, or NF1, compared with patients of the triple-WT subtype. As originally described in myeloid cells, engagement of CSF-1R via binding of its ligands leads to the activation of the ERK and PI3K/AKT pathways, promoting proliferation, differentiation, and survival (29). In melanoma, continuous BRAF V600E inhibition leads to a complete halt of signaling through the MAPK pathway, followed by the appearance of a resistant population displaying a rebound in ERK phosphorylation.

Our data show the timing of the rebound coinciding with increasing levels and maturation of the CSF-1R protein, as well as with higher expression of RUNX1 and IL-34. This rebound in ERK phosphorylation, at least in part, depends on the activity of the CSF-1R, since (a) the administration of PLX3397 completely blocks the phosphorylation of ERK and (b) the addition of CSF-1 or IL-34 further accelerates rebound from BRAF inhibition in the cells, which can be attenuated by cotreating the cells with the both the BRAF V600E and the CSF-1R inhibitor before the addition of the ligand. Coinhibition of BRAF and CSF-1R in the immunoblotting experiments was only possible for 2 hours; anything longer resulted in complete cell death (data not shown). ERK rebound has been found to be associated with upregulation of the PI3K/AKT pathway either via adaptive activation of receptor tyrosine kinases or by means of genetic alterations in the pathway $(47,48)$. Our studies 


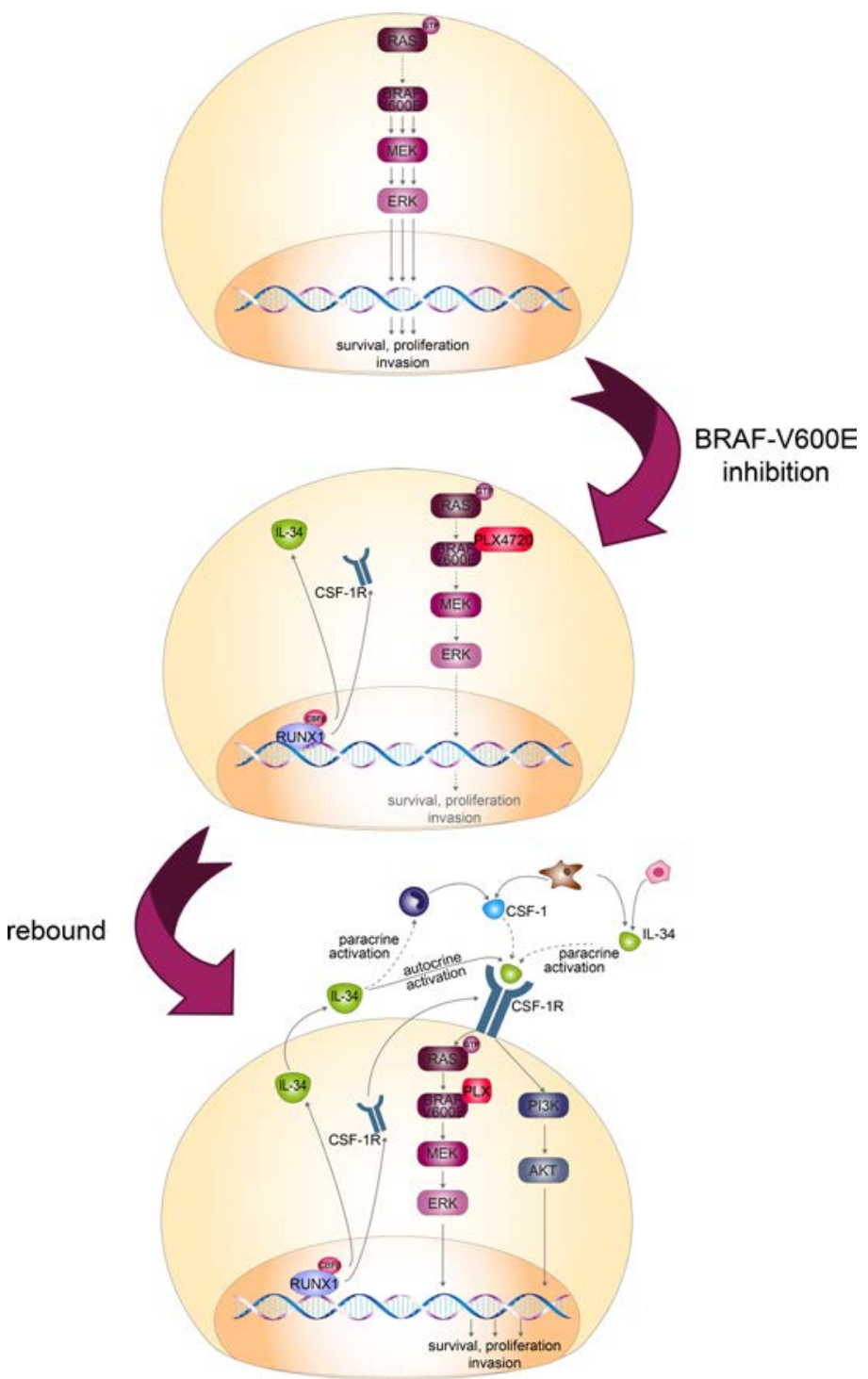

Figure 8. The role of CSF-1R and IL-34 in BRAF V600E inhibitor resistance. Low-level ERK-pathway activity induces the expression of RUNX1, leading to expression, maturation, and presentation of CSF-1R and IL-34. Coexpression of the receptor and the ligand leads to high oncogenic potential because of paracrine activation. The CSF-1R on the surface of melanoma cells can also be activated by ligands expressed by other cell types present in the microenvironment, and IL-34 can activate other cell types bearing the receptor in a paracrine manner.

demonstrate the involvement of CSF1R in the rebound via activation of the PI3K/AKT pathway, as well. PI3K signaling has been described to be low in melanomas, where activating BRAF or NRAS-mutations cause them to be addicted to the MAPK pathway. However, after acquiring resistance, increasing levels of phosphorylated AKT (seen in vitro and in clinical samples) indicate a shift toward reliance on PI3K/AKT signaling termed the "phospho-AKT rebound" $(47,49)$. We have also presented evidence that increasing levels of hosphor-AKT accompany the reemergence of hosphor-ERK. Our data demonstrates that CSF-1R inhibition can inhibit rebound of both ERK and PI3K/AKT pathways in BRAF-treated melanoma $(47,50)$

Taken together, our studies have important therapeutic implications, as we show that coadministering PLX3397 and PLX4720 has a synergistic effect in vitro, demonstrated by CI values of less than 1 in 3 different V600E melanoma cell lines. Further support for this finding stems from our 3-D Matrigel cell culture and A2058 mice xenograft studies, which show statistically significant reductions of colony size, tumor size, and growth rate when both inhibitors are used. We have shown evidence indicating that melanoma patients with refractory disease show increased levels of CSF1R, RUNX1, and IL-34, suggesting a role for these in clinical resistance. The question arising now is what the consequences of the coexpression of the receptor and its ligand would be in the context of the microenvironment of melanoma, which is already rich in cell types that can express one or both ligands of this receptor tyrosine kinase. Fibroblasts, tissue monocytes, and macrophages, as well as keratinocytes, have all been described to release CSF-1, while keratinocytes and fibroblasts are the main producers of IL-34 (51-53). Here, we have shown that melanoma 
cells themselves can also contribute to the pool of this latter cytokine. Coexpression of the CSF-1R and its ligand on the same cell results in great oncogenic potential, as the receptor can be constitutively activated due to an autocrine loop. Examining the known components of the tumor microenvironment leads us to believe that the receptors expressed on the surface of melanoma cells can also be activated in a paracrine manner by either ligand making for an even greater oncogenic potential, while the CSF-1Rs expressed on the TAMs can be engaged by the IL-34 secreted by the tumor cells, as well (Figure 8) $(51,54,55)$.

We propose that melanomas harboring the V600E mutation can acquire resistance by upregulating expression of both CSF-1R and IL-34, which (a) leads to activation of the PI3K/AKT and ERK pathways and (b) possibly contributes to an immunosuppressive, tumor permissive microenvironment $(51,54,55)$. Based on data presented in our study, we believe the IL-34/CSF-1R axis presents itself as an attractive, druggable target for clinical use and merits further investigation.

\section{Methods}

Reagents. PLX4720 and PLX3397 were provided by Plexxikon under a materials transfer agreement (MTA). Trametinib was purchased from Selleck Chemicals. Human recombinant CSF-1 and IL-34 was purchased from eBioscience.

Patient samples and nucleic acid extraction. Melanoma specimens were derived between 2004 and 2007, prior to the availability of any targeted treatment options. Snap-frozen samples were used for nucleic acid extraction. Genomic DNA was extracted by a standard phenol-chloroform protocol, followed by an ethanol precipitation and resuspension in $10 \mathrm{mM}$ Tris-HCl, $\mathrm{pH} 8.0$ (Thermo Fisher Scientific). Total RNA was extracted using the Qiagen RNeasy Mini kit and subjected to amplification using the MessageAmp II aRNA kit (Ambion). Reverse transcription from benign nevi RNA was performed with First-strand cDNA Synthesis (OriGene).

Cell culture conditions. All melanoma lines were maintained in DMEM supplemented with 10\% FBS (Thermo Fisher Scientific). U937 and THP-1 were maintained in RPMI (Hyclone) with 10\% heat-inactivated FBS (Gemini). Cells were cultured at $37^{\circ} \mathrm{C}$ in a humidified atmosphere of $5 \% \mathrm{CO}_{2}$ in air and harvested from subconfluent $(<80 \%$ ) cultures using a $0.05 \%$ trypsin-EDTA (Thermo Fisher Scientific) solution. Cell lines were used for up to 8 passages from thawing and were discarded after. All tissue cultures were tested for mycoplasma contamination on a regular basis using the InvivoGen PlasmoTest Mycoplasma Detection Kit. All cell lines were purchased from the American Type Tissue Collection (ATCC), except for the human melanoma cell line, M14c\#5, which was obtained as a gift from Barbara M. Mueller (La Jolla Institute for Molecular Medicine, La Jolla, California, USA). All cell line identities were authenticated using human STR profiling. Cultured normal foreskin-derived epidermal melanocyte (NHEM) control cells were obtained from the Vanderbilt University Translational Pathology Shared Resource Facility. Juvenile and adult melanocyte pellets from foreskin $(\mathrm{mc} 3 \mathrm{~W}, \mathrm{mc} 2 \mathrm{~W})$ and adult melanocyte pellets from facial skin (mc32W, mc65W) for DNA and RNA work were purchased from PromoCell. RNA from the cell lines was extracted using the Purelink RNA Mini kit (Ambion) with on-column DNase treatment with the Qiagen RNase-Free DNase Set, as per manufacturer's protocol.

DNA methylation analysis by HELP assay. The HELP assay was carried out as previously published (56) Intact DNA of high molecular weight was corroborated by electrophoresis on $1 \%$ agarose gel in all cases. Genomic DNA $(1 \mu \mathrm{g})$ was digested overnight with either HpaII or MspI (New England Biolabs). On the following day, the reactions were extracted once with phenol-chloroform and resuspended in $11 \mu 1$ of $10 \mathrm{mM}$ Tris- $\mathrm{HCl}, \mathrm{pH} 8.0$, and the digested DNA was used to set up an overnight ligation of the JHpaII adapter using T4 DNA ligase. The adapter-ligated DNA was used to carry out the PCR amplification of the HpaII- and MspI-digested DNA as previously described (56). Both amplified fractions were submitted to Roche-NimbleGen Inc. for labeling and hybridization onto a human hg17 custom-designed oligonucleotide array (50mers) covering 25,626 HpaII amplifiable fragments (HAF) located at gene promoters. HAFs are defined as genomic sequences contained between 2 flanking HpaII sites found within 200-2,000 bp from each other. Each fragment on the array is represented by 15 individual probes distributed randomly spatially across the microarray slide. Thus, the microarray covers 50,000 CpGs corresponding to 14,000 gene promoters.

HELP data processing and analysis. Signal intensities at each HAF were calculated as a robust ( $25 \%$ trimmed) mean of their component probe-level signal intensities. Any fragments found within the level of background MspI signal intensity, measured as 2.5 mean absolute differences (MAD) above the median of random probe signals, were categorized as "failed." These "failed" loci, therefore, represent the population of fragments not amplified by PCR, whatever the biological (e.g., genomic deletions and other sequence errors) or experimen- 
tal cause. On the other hand, "methylated" loci were so designated when the level of HpaII signal intensity was similarly indistinguishable from background. PCR-amplifying fragments (those not flagged as either "methylated" or "failed") were normalized using an intraarray quantile approach wherein HpaII/MspI ratios are aligned across density-dependent sliding windows of fragment size-sorted data. The log HpaII/MspI ratio was used as a representative for methylation and analyzed as a continuous variable. For most loci, each fragment was categorized as either methylated, if the centered log HpaII/MspI ratio was less than zero, or hypomethylated if - on the other hand - the log ratio was greater than zero.

Quantitative DNA methylation analysis by Mass ARRAY epityping. Validation of HELP microarray findings was carried out by matrix-assisted laser desorption/ionization-TOF (MALDI-TOF) mass spectrometry using EpiTYPER Methylation Analysis (Agena Bioscience). Genomic DNA was bisulfite converted with the EZ DNA Methylation-Direct Kit (Zymo Research), then 10-100 ng was amplified with PCR using the Roche FastStart High Fidelity PCR System. MassARRAY primers (Supplemental Table 5) were designed to cover the flanking HpaII sites for a given HAF, as well as any other HpaII sites found up to 2,000 bp upstream of the downstream site and up to 2,000 bp downstream of the upstream site, in order to cover all possible alternative sites of digestion. Gel-purified amplicons were used as input, and results were visualized with the EpiTYPER software.

Microarray quality control. All microarray hybridizations were subjected to extensive quality control using the following strategies. First, uniformity of hybridization was evaluated using a modified version of a previously published algorithm $(57,58)$ adapted for the NimbleGen platform, and any hybridization with strong regional artifacts was discarded and repeated. Second, normalized signal intensities from each array were compared against a $20 \%$ trimmed mean of signal intensities across all arrays in that experiment, and any arrays displaying a significant intensity bias that could not be explained by the biology of the sample were excluded.

Microarray data analysis. Unsupervised clustering of HELP data by hierarchical clustering was performed on the 29 samples, with a Ward linkage function and distance between samples calculated as 1 - abs(cor[x,y]) over the full set of 17,049 log probe ratios, with NA values removed. The clustering dendrogram shows 4 clear-cut groups distinguishable by height threshold and was combined with a heatmap of 500 randomly selected probes in a composite figure. Volcano plots were constructed looking at the interesting pairwise combinations of the 4 groups as defined by the clustering dendrogram. Samples were split into 2 groups, and at each loci, a $t$ test was run. The results of each loci are displayed as a 2-D point, with the $x$ axis showing the mean difference of $\log$ ratio values and the $y$ axis showing the $t$ test's $-\log _{10} P$ value. Loci with a mean difference greater than 1 and $P<0.01$ are shown.

Array-based comparative genomic hybridization. Gene copy number changes were analyzed by high-resolution $(6 \mathrm{~kb})$ microarray-based comparative genomic hybridization (aCGH) performed on Roche-NimbleGen 385K whole genome tiling arrays (2006-11-01_HG17_WG_CGH). Pooled DNA from healthy cases was used as a control during hybridization. These arrays contain 50-75 mer probes at average spacing of 6,270 bp (6 kb). These probe-level aCGH data were analyzed by DNA copy algorithm (Nimblescan software package, Roche-NimbleGen Inc.) using 5 adjacent oligonucleotides and confirmed by circular binary segmentation algorithm. Significant DNA copy number changes were cross referenced from the HapMap database from NCBI to remove normal variants.

Pathway and transcription factor binding site analysis. We carried out an analysis of the biological information retrieved by each of the individual platforms alone and compared it to the information obtained by the integrated analysis of all 3 platforms. Enrichment of genes associated with specific canonical pathways was determined relative to the ingenuity knowledge database IPA software (Qiagen) at a significance level of $P<0.01$. The list of differentially methylated genes was examined for enrichment of conserved gene-associated transcription factor binding sites using the Molecular Signatures Database (MSigDB) (http:// software.broadinstitute.org/gsea/msigdb/).

LUMA. Genomic DNA (200-500 ng) was cleaved with HpaII + EcoRI or MspI + EcoRI in 2 separate $20-\mathrm{ml}$ reactions, and the assay was performed as described previously. Samples were run in a PSQ 96MA system (Biotage AB) programmed to add dNTPs in 4 consecutive steps: step 1, dATP (the derivative dAT$\mathrm{P} \alpha \mathrm{S}$ is used, since it will not react directly with luciferase and prevents nonspecific signals); step 2, mixture of dGTP + dCTP; step 3, dTTP; and step 4, mixture of dGTP + dCTP. Peak heights were calculated using the PSQ 96MA software. The HpaII/EcoRI and MspI/EcoRI ratios were calculated as (dGTP + dCTP)/dATP for the respective reactions. The HpaII/MspI ratio was defined as (HpaII/EcoRI)/(MspI/EcoRI) (56, 59). 
Quantification of global DNA hydroxymethylation $(\mathrm{HmC})$ and methylation $(\mathrm{mC})$ by mass spectrometry. An selected reaction monitoring-based (SRM-based) mass spectrometry assay was used to quantify 5-hydroxymethyl-2'-deoxycytidine $(5 \mathrm{HmdC})$ and 5-methyl-2'-deoxycytidine $(5 \mathrm{mdC})$. The assay was designed to measure $5 \mathrm{HmdC}$ concentrations and $5 \mathrm{mdC}$ concentrations as a percentage of 2 '-deoxyguanosine (dG) (e.g., $-[5 \mathrm{HmdC}] /[\mathrm{dG}]$ and $[5 \mathrm{mdC}] /[\mathrm{dG}])$. The calibrated ranges for the analytes were $0 \%-2.5 \%$ for $5 \mathrm{HmdC}$ and $0 \%-25 \%$ for $5 \mathrm{mdC}$ using a fixed 40 -pmol amount of $\mathrm{dG}$ as an internal standard. The calibration points were run as single replicates due to previously demonstrated high reproducibility of the assay. All the procedures were carried out by Zymo Research Epigenetic Services (Zymo Research Corp.).

IHC on melanoma tissue microarray. Archival formalin-fixed paraffin-embedded melanoma tissue samples were used to construct 4 tissue microarrays (TMAs) with at least 4 replicates per block. TMAs were stained for MelanA/MART1 (commonly used melanocytic marker) using Bond Ready-to-Use Primary Antibody Melan A (A103) from Leica or, for CSF-1R, using an antibody from E. Richard Stanley (Albert Einstein College of Medicine) raised in rabbits against the peptide [C]-GDIAQPLLQPNNYQF-962-976 and affinity-purified against their corresponding peptides (60). Semiquantitative assessment of CSF1R antibody staining on the TMAs was performed by a pathologist who was blinded to the identities of the tissues. Staining was compared with a positive control (placenta). The maximal intensity of vector red staining was graded on a $0-3$ scale ( 0 , negative; $1+$, weak; $2+$, moderate; $3+$, strong staining).

RNA-FISH. Cells of melanocytic origin were paraffin embedded and mounted onto glass slides. RNA-expression was investigated by hybridizing complementary oligonucleotide probes attached to fluorescent dyes using the RNAscope LS Multiplex Fluorescent Reagent Kit (Advanced Cell Diagnostics) as per the manufacturer's recommendations. For all probes used, see Supplemental Table 5. All the procedures were carried out by ACD Support RNAscope Pilot Study Service.

$q P C R$ and sequence verification. cDNA was prepared with the SuperScript III Reverse Transcriptase kit (Invitrogen). qPCR reactions were carried out using the VeriQuest Fast SYBR Green qPCR MasterMix with Fluorescein (Affymetrix) or TaqMan Fast Advanced Mastermix (Invitrogen) on a Bio-Rad CFX96 instrument. All PCR primers were designed with the aid of PerlPrimer (61), with 1 primer spanning an intron-exon junction when appropriate and when possible. The $\mathrm{qPCR}$ primer efficiencies were all kept between $95 \%-105 \%$ and were considered in the calculation of relative expression values. The aberrant CSF1R gene product (LTR) was verified by synthesizing CDNA using a gene-specific primer and then amplifying, gel-purifying from agarose, and sequencing a 522-bp PCR product containing the unique $5^{\prime}$-UTR. For all primer (IDT) and probe (Applied Biosystems) sequences, see Supplemental Table 6. GAPDH was used as housekeeper gene for all qPCR experiments.

Three-dimensional culture of melanoma cells. Three-dimensional laminin-rich extracellular matrix cultures (62) were prepared by trypsinization of melanoma cells from tissue culture plastic, the seeding of single cells on top of a thin layer of Growth Factor-Reduced Matrigel (BD Biosciences), and the addition of a medium containing $5 \%$ Matrigel. The cells were seeded at the density of 40,000 cells per $\mathrm{cm}^{2}$. All 3-D cell cultures were performed in DMEM supplemented with $5 \mu \mathrm{g} / \mathrm{ml}$ bovine insulin with Zinc (Thermo Fisher Scientific), $15 \mu \mathrm{g} / \mathrm{ml}$ bovine pituitary extract (Thermo Fisher Scientific), $0.5 \mathrm{ng} / \mathrm{ml}$ epidermal growth factor (MilliporeSigma), and 2\% FBS. Three-dimensional cultures were maintained with media changes every second day, and photographs were taken at the end of the fifth day. The size of single cell colonies was estimated with ImageJ (NIH) (63).

Proliferation and apoptosis assays. BrdU labeling and TUNEL assay of the 3-D colonies were carried out as described previously (64).

Generation of stable cell lines. Stable suppression of CSF1R and RUNX1 expression was achieved via lentiviral transduction. A nontargeting shRNA was used to control for off-target effects. To produce the lentivirus, pGIPZ plasmids (Dharmacon) were cotransfected into HEK293T cells with the following helper plasmids: pRc/CMV, pHDM-Hgpm2, pHDM-tat1b, and pHDM-VSV-G, using Effectene transfection reagent (Qiagen). For lentiviral transduction, melanoma cells were seeded 16 hours before and infected with a 1:6 dilution of fresh viral supernatant with $8 \mu \mathrm{g} / \mathrm{ml}$ Polybrene (Santa Cruz Biotechnology Inc.). Puromycin (Thermo Fisher Scientific) was used to select for stable clones of melanoma cells harboring the appropriate shRNA. Knockdown efficiency was confirmed with qPCR (Figure 2, A and B). shCSF1R clones 1 and 5 and shRUNX1 clones 1 and 4 were validated as the best shRNAs to be used, as each demonstrated highest knockdown efficiency and no off-target effects. For clone IDs and sense sequences, see Supplemental Table 6.

To generate promoter-reporter gene fusions, the pGL4.19[lucCP/Neo] (Promega) vector was used. For the viral LTR construct, chr5:148,904,955-148,904,605 was cloned into the multiple cloning site of the vector 
(pGL4.19-LTR). For the construct containing the canonical promoter of CSF1R, we cloned chr5:150,114,454150,113,121 into the MCS. Melanoma cell lines stably expressing CSF1R promoter-luciferase constructs were generated via transfection with Effectene and selection with G418-Sulfate (Thermo Fisher Scientific).

Matrigel invasion assay. The invasiveness of the A2058 cells were assessed with the modified Boyden chamber assay. BD BioCoat Matrigel Invasion Chambers with $8 \mu \mathrm{m}$ pore sizes in a 24 -well plate format were used, per the manufacturer's recommendation. After the cells were allowed to invade, the Matrigel was wiped off the membrane; then, it was fixed with $4 \%$ paraformaldehyde and stained with $0.2 \%$ crystal violet (both from Thermo Fisher Scientific), and the number of cells that had invaded through to the other side of the membrane were counted.

ELISA. Cultured cells were allowed to condition media for 48 hours. The supernatant was collected and centrifuged at full speed to eliminate cell debris. It was then frozen and stored in single-use aliquots at $-80^{\circ} \mathrm{C}$. The cell numbers were counted and used for normalizing the measured protein concentrations. Secreted cytokines were quantified using the human IL-34 and the human M-CSF DuoSet ELISA Development kits (R\&D Systems) according to the manufacturer's instructions.

Immunoblotting and flow cytometry cell analysis. Cells were lysed with ice-cold $1 \times$ RIPA-buffer (Cell Signaling Technologies) containing $1 \times$ Halt Protease and Phosphatase Inhibitor Cocktail (Thermo Fisher Scientific). Protein amounts were determined with the BCA Protein Assay (Pierce), and $25 \mu \mathrm{g}$ of each was used for immunoblot analysis. Samples were loaded onto a 4-12\% Bolt Bis-Tris gel (Invitrogen) and, after separation, were transferred to a polyvinylidene difluoride membrane (Bio-Rad). The membranes were blocked and incubated with the following primary antibodies: phospho-p44/42 MAPK (Erk1/2, Thr202/ Tyr204, catalog 9101), p44/42 MAPK (Erk1/2, catalog 9102), Phospho-Akt (Ser473, catalog 9271), and AKT (catalog 9272) (from Cell Signaling Technologies) or CSF-1R (sc-46662) and Actin HRP (catalog sc-1615) (catalog Santa Cruz Biotechnologies Inc.) as loading control. Primary antibodies were followed by incubation with horseradish peroxidase-conjugated anti-mouse (Santa Cruz Biotechnologies Inc., catalog sc-358914) or anti-rabbit secondary antibodies (Cell Signalling Technologies, catalog7074), and the blots were developed with the Western Lightning Plus-ECL system (Perkin-Elmer). For FACS-analysis, the M-CSF Receptor (PE Conjugate) antibody (Cell Signalling Technologies, 65396) was used.

Cytotoxicity assay. Cell viability was determined with a resazurin-based assay. Four thousand (A2058 or WM-266-4 cell lines) or 2,000 cells (M14C\#5) were seeded in $100 \mu 1$ DMEM with 10\% FBS in a 96-well plate. After 24 hours, the media was replaced with full growth medium containing the appropriate drug concentration and incubated with the cells for a total of 72 hours of treatment. The medium was then fully replaced with fresh serum-free DMEM with $10 \%$ alamarBlue (Bio-Rad), the cells were incubated for 60 minutes at $37^{\circ} \mathrm{C}$, and signal $\left(\lambda_{\text {exc }}=570 \mathrm{~nm}, \lambda_{\text {em }}=590 \mathrm{~nm}\right)$ was measured on a FLUOStar Omega multiwell fluorimeter. After baseline (no cell control) adjustment, the measured signal was normalized to the DMSO-treated cells designated to be $100 \%$ viable. Nonlinear regression in GraphPad Prism 7 was utilized to fit the growth curve over the various drug concentrations to determine the half-maximal inhibitory concentration $\left(\mathrm{IC}_{50}\right), \mathrm{IC}_{40}$, and $\mathrm{IC}_{75}$, etc.

The drug combination experiments were conducted using constant and nonconstant ratios of the therapeutic agents based on the $\mathrm{IC}_{50}$ value of each drug. Using a constant ratio, PLX4720 (A2058 $\mathrm{IC}_{50}=0.332$ $\mu \mathrm{M})$ was combined with either PLX3397 (A2058 $\left.\mathrm{IC}_{50}=19.573 \mu \mathrm{M}\right)$ or Trametinib $\left(\mathrm{A} 2058 \mathrm{IC}_{50}=1.386\right.$ $\mathrm{nM}$ ) and serially diluted. In another set of experiments, the nonfixed ratio method was used. PLX3397 was maintained at $10 \mu \mathrm{M}$ or $15 \mu \mathrm{M}$ concentration and combined with serially diluted PLX4720 (cell lines tested: WM-266-4 and M14c\#5). In both methods, melanoma cells were treated in quadruplicates for 72 hours, and cell growth inhibition was estimated by the alamarBlue assay. The Chou-Talalay method was used to determine the $\mathrm{CI}$, where $\mathrm{CI}=1$ refers to additive effect, $\mathrm{CI}<1$ means synergism, and $\mathrm{CI}>1$ refers to antagonism in drug combinations (34).

Xenografting studies. Female BALB/c nude mice, 5-6 weeks old, were inoculated s.c. with A2058 tumor fragments $(2 \mathrm{~mm} \times 2 \mathrm{~mm} \times 2 \mathrm{~mm})$ on the right flank. After 11 days, when the mean tumor size reached $140 \mathrm{~mm}^{3}$, groups of 10 mice each were given daily administrations of $20 \mathrm{mg} / \mathrm{kg}$ PLX3397, $20 \mathrm{mg} / \mathrm{kg}$ PLX4720, both drugs combined, or 1\% carboxymethylcellulose/10\% DMSO as vehicle control. Mice were euthanized when tumors reached 2,500 $\mathrm{mm}^{3}$, termed "death" to calculate survival curves.

Statistics. Previously published patient RNA-seq datasets were used to examine expression changes in our genes of interest after patients showed either stable disease or partial response to drug treatment.

Bam files were downloaded with permission and were processed with HTSeq-count version 0.6.1p1 (65) using the GRCh37.75 gene set annotation file and default parameters to obtain gene read counts. The 
union overlap scheme was used to resolve reads appearing in more than 1 gene. Gene read counts were normalized with the DESeq R package version 1.20.0 (66) by dividing each sample's counts by its size factor. Count ratios were calculated by dividing each column (sample) by the geometric means across rows (gene). A sample's size factor is the median of these ratios over all genes. The TCGA melanoma gene expression, mutation and methylation data were downloaded from CBioPortal $(67,68)$. All other data analysis was performed using GraphPad Prism 7. All data are available in the GEO database (https://www.ncbi.nlm. nih.gov/geo/) under accession GSE97457.

Study approval. Melanoma specimens were obtained from patients diagnosed with melanoma and controls. Study was approved by the Vanderbilt Human Research Protections Program/IRB under IRB 100178. Approval to collect benign nevi was granted by ISMMS (Icahn School of Medicine at Mount Sinai) Division of Dermatopathology (project number 08-0964). All specimen were collected after signed informed consent prior to study entry.

\section{Author contributions}

$\mathrm{OG}$ and AKV conceived and designed the research. YM performed the HELP assay, array CGH, and MicroArray experiments. OG, YM, and MB performed the MassARRAY EpiTYPER assay. OG performed all qPCR analyses, molecular cloning, and stable cell line creation, as well as all cell line-based in vitro experiments and immunoblotting. VP contributed to RNA extraction. XMCR contributed to the immunoblotting. TDB contributed to nucleic acid extraction. HN, BM, RS, EB, JT, and CZ performed the in vivo mouse experiments and alternative transcript sequencing experiments. KBD prepared, analyzed, and quantified the histology. DBJ oversaw the clinical data. KP and YY performed the bioinformatic analyses. OG, KP, and YY performed the statistical analyses. OG analyzed the data. OG and AV wrote the manuscript. All coauthors edited the manuscript. Data and materials availability: all data for this study are presented here, and all material is available for noncommercial researchers via a MTA.

\section{Acknowledgments}

We thank the Albert Einstein Genomics Core, David Reynolds and Kevin Lau for help with the MassARRAY EPITYPER assay. Part of this work was supported by the Pershing Square Sohn Cancer Research Alliance and Department of Defense W81XWH-14-1-0230 to EB. The RNA in situ hybridization slides were scanned by Vera DesMarais in the Analytical Imaging Facility at Albert Einstein College of Medicine, which is funded by Cancer Center grant P30CA013330, using the 3DHistec Panoramic 250 Flash II slide scanner (funded by SIG 1S10OD019961-01).

Address for correspondence: Amit Verma or Orsi Giricz, Albert Einstein College of Medicine, 1300 Morris Park Avenue, Chanin302B, Bronx, New York 10461, USA. Phone: 718.430.8761; Email: amit.verma@ einstein.yu.edu (A. Verma). Phone: 718.430.2786; Email: orsi.giricz@gmail.com (O. Giricz).

JAS's present address is: Robert Lurie Cancer Canter, Northwestern University, Chicago, Illinois, USA.

1. Howlader N, et al. SEER Cancer Statistics Review, (CSR) 1975-2015. National Cancer Institute. https://seer.cancer.gov/ csr/1975_2015/. Published April 16, 2018. Accessed June 27, 2018.

2. Cancer Genome Atlas Network. Genomic Classification of Cutaneous Melanoma. Cell. 2015;161(7):1681-1696.

3. Eroglu Z, Ribas A. Combination therapy with BRAF and MEK inhibitors for melanoma: latest evidence and place in therapy. Ther Adv Med Oncol. 2016;8(1):48-56.

4. Flaherty KT, et al. Improved survival with MEK inhibition in BRAF-mutated melanoma. N Engl J Med. 2012;367(2):107-114.

5. Wolchok JD, et al. Updated results from a phase III trial of nivolumab (NIVO) combined with ipilimumab (IPI) in treatment-naive patients (pts) with advanced melanoma (MEL) (CheckMate 067). J Clin Oncol. 2016;34(15_suppl):9505.

6. Zaretsky JM, et al. Mutations Associated with Acquired Resistance to PD-1 Blockade in Melanoma. $N$ Engl J Med. 2016;375(9):819-829.

7. Lee JJ, Murphy GF, Lian CG. Melanoma epigenetics: novel mechanisms, markers, and medicines. Lab Invest. 2014;94(8):822838.

8. Lauss $\mathrm{M}$, et al. DNA methylation subgroups in melanoma are associated with proliferative and immunological processes. $B M C$ Med Genomics. 2015;8:73.

9. Li JL, et al. Genome-wide methylated CpG island profiles of melanoma cells reveal a melanoma coregulation network. Sci Rep 2013;3:2962.

10. Shaknovich R, Figueroa ME, Melnick A. HELP (HpaII Tiny Fragment Enrichment by Ligation-Mediated PCR) Assay for DNA Methylation Profiling of Primary Normal and Malignant B Lymphocytes. In: Chittur SV, ed. Microarray Methods for Drug 
Discovery. Totowa, NJ: Humana Press; 2010:191-201.

11. Suzuki M, Greally JM. DNA methylation profiling using HpaII tiny fragment enrichment by ligation-mediated PCR (HELP). Methods. 2010;52(3):218-222.

12. Irizarry RA, et al. The human colon cancer methylome shows similar hypo- and hypermethylation at conserved tissue-specific CpG island shores. Nat Genet. 2009;41(2):178-186.

13. Jones PA, Gonzalgo ML. Altered DNA methylation and genome instability: a new pathway to cancer? Proc Natl Acad Sci USA 1997;94(6):2103-2105.

14. Bröcker EB, Zwadlo G, Holzmann B, Macher E, Sorg C. Inflammatory cell infiltrates in human melanoma at different stages of tumor progression. Int J Cancer. 1988;41(4):562-567.

15. Nandi S, et al. The CSF-1 receptor ligands IL-34 and CSF-1 exhibit distinct developmental brain expression patterns and regulate neural progenitor cell maintenance and maturation. Dev Biol. 2012;367(2):100-113.

16. Zhang DE, et al. CCAAT enhancer-binding protein (C/EBP) and AML1 (CBF alpha2) synergistically activate the macrophage colony-stimulating factor receptor promoter. Mol Cell Biol. 1996;16(3):1231-1240.

17. Lamprecht B, et al. Derepression of an endogenous long terminal repeat activates the CSF1R proto-oncogene in human lymphoma. Nat Med. 2010;16(5):571-579.

18. Haltaufderhyde KD, Oancea E. Genome-wide transcriptome analysis of human epidermal melanocytes. Genomics. 2014;104(6 Pt B):482-489.

19. Sandelin A, Wasserman WW, Lenhard B. ConSite: web-based prediction of regulatory elements using cross-species comparison. Nucleic Acids Res. 2004;32(Web Server issue):W249-W252.

20. DeNardo DG, et al. Leukocyte complexity predicts breast cancer survival and functionally regulates response to chemotherapy. Cancer Discov. 2011;1(1):54-67.

21. Tap WD, et al. Structure-Guided Blockade of CSF1R Kinase in Tenosynovial Giant-Cell Tumor. N Engl J Med. 2015;373(5):428-437.

22. Lito P, et al. Relief of profound feedback inhibition of mitogenic signaling by RAF inhibitors attenuates their activity in BRAFV600E melanomas. Cancer Cell. 2012;22(5):668-682.

23. Sun C, et al. Reversible and adaptive resistance to BRAF(V600E) inhibition in melanoma. Nature. 2014;508(7494):118-122.

24. Nazarian R, et al. Melanomas acquire resistance to B-RAF(V600E) inhibition by RTK or N-RAS upregulation. Nature. 2010;468(7326):973-977.

25. Poulikakos PI, Rosen N. Mutant BRAF melanomas--dependence and resistance. Cancer Cell. 2011;19(1):11-15.

26. Moriceau G, et al. Tunable-combinatorial mechanisms of acquired resistance limit the efficacy of BRAF/MEK cotargeting but result in melanoma drug addiction. Cancer Cell. 2015;27(2):240-256.

27. Hamilton JA. CSF-1 signal transduction. J Leukoc Biol. 1997;62(2):145-155.

28. Pixley FJ, Stanley ER. CSF-1 regulation of the wandering macrophage: complexity in action. Trends Cell Biol. 2004;14(11):628-638.

29. Stanley ER, Chitu V. CSF-1 receptor signaling in myeloid cells. Cold Spring Harb Perspect Biol. 2014;6(6):a021857.

30. Tsai J, et al. Discovery of a selective inhibitor of oncogenic B-Raf kinase with potent antimelanoma activity. Proc Natl Acad Sci USA. 2008;105(8):3041-3046.

31. Bollag G, et al. Clinical efficacy of a RAF inhibitor needs broad target blockade in BRAF-mutant melanoma. Nature. 2010;467(7315):596-599.

32. Van Allen EM, et al. The genetic landscape of clinical resistance to RAF inhibition in metastatic melanoma. Cancer Discov. 2014;4(1):94-109.

33. Kwong LN, et al. Co-clinical assessment identifies patterns of BRAF inhibitor resistance in melanoma. J Clin Invest. 2015;125(4):1459-1470.

34. Chou TC. Theoretical basis, experimental design, and computerized simulation of synergism and antagonism in drug combination studies. Pharmacol Rev. 2006;58(3):621-681.

35. Micevic G, Theodosakis N, Taube JM, Bosenberg MW, Rodić N. Attenuation of genome-wide 5-methylcytosine level is an epigenetic feature of cutaneous malignant melanomas. Melanoma Res. 2017;27(2):85-96.

36. Ide $\mathrm{H}$, et al. Expression of colony-stimulating factor 1 receptor during prostate development and prostate cancer progression. Proc Natl Acad Sci USA. 2002;99(22):14404-14409.

37. Tang R, Beuvon F, Ojeda M, Mosseri V, Pouillart P, Scholl S. M-CSF (monocyte colony stimulating factor) and M-CSF receptor expression by breast tumour cells: M-CSF mediated recruitment of tumour infiltrating monocytes? J Cell Biochem 1992;50(4):350-356.

38. Menke J, Kriegsmann J, Schimanski CC, Schwartz MM, Schwarting A, Kelley VR. Autocrine CSF-1 and CSF-1 receptor coexpression promotes renal cell carcinoma growth. Cancer Res. 2012;72(1):187-200.

39. Laoui D, Van Overmeire E, De Baetselier P, Van Ginderachter JA, Raes G. Functional Relationship between Tumor-Associated Macrophages and Macrophage Colony-Stimulating Factor as Contributors to Cancer Progression. Front Immunol. 2014;5:489.

40. Mok S, et al. Inhibition of colony stimulating factor-1 receptor improves antitumor efficacy of BRAF inhibition. BMC Cancer. 2015; $15: 356$.

41. Sluijter M, et al. Inhibition of CSF-1R supports T-cell mediated melanoma therapy. PLoS ONE. 2014;9(8):e104230.

42. Ngiow SF, et al. Co-inhibition of colony stimulating factor-1 receptor and BRAF oncogene in mouse models of BRAFV600E melanoma. Oncoimmunology. 2016;5(3):e1089381.

43. Ryder M, et al. Genetic and pharmacological targeting of CSF-1/CSF-1R inhibits tumor-associated macrophages and impairs BRAF-induced thyroid cancer progression. PLoS One. 2013;8(1):e54302.

44. Benci JL, et al. Tumor Interferon Signaling Regulates a Multigenic Resistance Program to Immune Checkpoint Blockade. Cell. 2016;167(6):1540-1554.e12.

45. Borycki AG, Smadja F, Stanley R, Leibovitch SA. Colony-stimulating factor 1 (CSF-1) is involved in an autocrine growth control of rat myogenic cells. Exp Cell Res. 1995;218(1):213-222.

46. Hugo W, et al. Non-genomic and Immune Evolution of Melanoma Acquiring MAPKi Resistance. Cell. 2015;162(6):1271-1285.

47. Shi H, et al. A novel AKT1 mutant amplifies an adaptive melanoma response to BRAF inhibition. Cancer Discov. 
2014;4(1):69-79.

48. Shi H, et al. Acquired resistance and clonal evolution in melanoma during BRAF inhibitor therapy. Cancer Discov. 2014;4(1):80-93.

49. Villanueva J, et al. Acquired resistance to BRAF inhibitors mediated by a RAF kinase switch in melanoma can be overcome by cotargeting MEK and IGF-1R/PI3K. Cancer Cell. 2010;18(6):683-695.

50. Obenauf AC, et al. Therapy-induced tumour secretomes promote resistance and tumour progression. Nature. 2015;520(7547):368-372.

51. Wang T, et al. Melanoma-derived conditioned media efficiently induce the differentiation of monocytes to macrophages that display a highly invasive gene signature. Pigment Cell Melanoma Res. 2012;25(4):493-505.

52. Lin $\mathrm{H}$, et al. Discovery of a cytokine and its receptor by functional screening of the extracellular proteome. Science. 2008;320(5877):807-811.

53. Wei S, et al. Functional overlap but differential expression of CSF-1 and IL-34 in their CSF-1 receptor-mediated regulation of myeloid cells. J Leukoc Biol. 2010;88(3):495-505.

54. Foucher ED, et al. IL-34 induces the differentiation of human monocytes into immunosuppressive macrophages. antagonistic effects of GM-CSF and IFN $\gamma$. PLoS One. 2013;8(2):e56045

55. Baghdadi M, et al. Chemotherapy-Induced IL34 Enhances Immunosuppression by Tumor-Associated Macrophages and Mediates Survival of Chemoresistant Lung Cancer Cells. Cancer Res. 2016;76(20):6030-6042.

56. Alvarez H, et al. Widespread hypomethylation occurs early and synergizes with gene amplification during esophageal carcinogenesis. PLoS Genet. 2011;7(3):e1001356.

57. Thompson RF, et al. An analytical pipeline for genomic representations used for cytosine methylation studies. Bioinformatics. 2008;24(9):1161-1167.

58. Khulan B, et al. Comparative isoschizomer profiling of cytosine methylation: the HELP assay. Genome Res. 2006;16(8):1046-1055.

59. Karimi M, et al. LUMA (LUminometric Methylation Assay)--a high throughput method to the analysis of genomic DNA methylation. Exp Cell Res. 2006;312(11):1989-1995.

60. Yu W, et al. CSF-1 receptor structure/function in MacCsf1r-/- macrophages: regulation of proliferation, differentiation, and morphology. J Leukoc Biol. 2008;84(3):852-863.

61. Marshall OJ. PerlPrimer: cross-platform, graphical primer design for standard, bisulphite and real-time PCR. Bioinformatics. 2004;20(15):2471-2472.

62. Lee GY, Kenny PA, Lee EH, Bissell MJ. Three-dimensional culture models of normal and malignant breast epithelial cells. Nat Methods. 2007;4(4):359-365.

63. Schneider CA, Rasband WS, Eliceiri KW. NIH Image to ImageJ: 25 years of image analysis. Nat Methods. 2012;9(7):671-675.

64. Giricz O, Calvo V, Pero SC, Krag DN, Sparano JA, Kenny PA. GRB7 is required for triple-negative breast cancer cell invasion and survival. Breast Cancer Res Treat. 2012;133(2):607-615

65. Anders S, Pyl PT, Huber W. HTSeq--a Python framework to work with high-throughput sequencing data. Bioinformatics. 2015;31(2):166-169.

66. Anders S, Huber W. Differential expression analysis for sequence count data. Genome Biol. 2010;11(10):R106.

67. Gao J, et al. Integrative analysis of complex cancer genomics and clinical profiles using the cBioPortal. Sci Signal. 2013;6(269):p11.

68. Cerami E, et al. The cBio cancer genomics portal: an open platform for exploring multidimensional cancer genomics data. Cancer Discov. 2012;2(5):401-404. 